\title{
Computational Porous Media Modeling of a Brush Seal for Incompressible and Slightly Compressible Flow
}

\author{
Thomas Gordon Gresham \\ Charlottesville, Virginia
}

B.S. Mechanical Engineering, University of Virginia, 2014

\begin{abstract}
A Thesis presented to the Graduate Faculty of the University of Virginia in Candidacy for the Degree of Master of Science

Department of Mechanical and Aerospace Engineering
\end{abstract}

University of Virginia

August, 2016 


\begin{abstract}
Annular seals are critical to the efficient operation of turbomachinery under increasingly high pressure loadings. Brush seals employ rows of bristles to close the gap between the rotor and the stator in rotating machinery. They typically handle larger pressure differentials and have demonstrated lower leakage than comparable labyrinth seals. However, an understanding of the complex nature of the flow within these seals is limited. This is primarily due to the difficulty associated with modeling the flow through the bristle pack.

Traditionally, the performance modeling of annular seals has been accomplished by the use of bulk flow theory. This method is relatively simple to implement but can be problematic due to the fundamental assumptions required to reduce the governing equations to a solvable form. More recent studies have used full three-dimensional computational fluid dynamics models and finite element models to simulate the behavior of the bristles and their influence on the flow through the seal. This method typically provides more information than is necessary for standard design and analysis problems faced by engineers in the turbomachinery industry. Simulations of this type also require a great deal of time and knowledge from the user in order to be of any use and are very computationally expensive. Porous media modeling techniques have also proven effective, but their application has remained limited due to the uncertainty of how to estimate the permeability terms and a lack of a simple computer code appropriate for the problem.

In this thesis, a new approach is presented using a simplified porous media-based model. The governing equations are formulated in terms of pressure distribution and then discretized using finite differences. An algorithm is developed to model situations of incompressible flow as well as compressible flow under isothermal conditions. The code is then analyzed to demonstrate its performance capabilities and utility for modeling flow through a standard brush seal. Properties of the numerical scheme are demonstrated and potential limitations are addressed.
\end{abstract}




\section{Contents}

\section{Introduction}

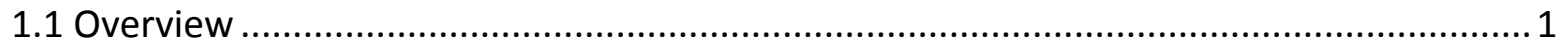

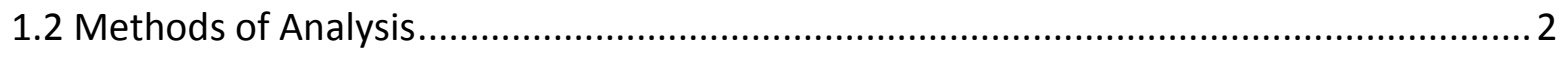

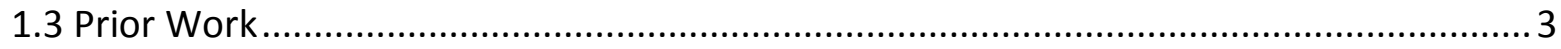

2 Seal Performance Modeling $\quad 5$

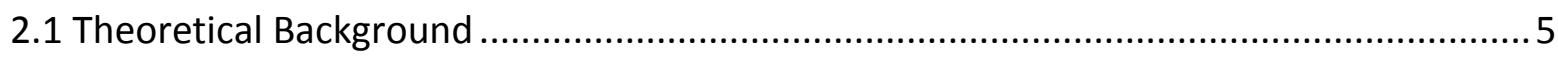

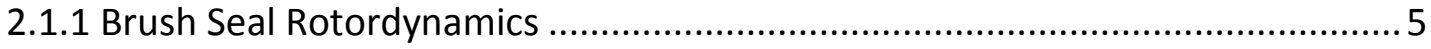

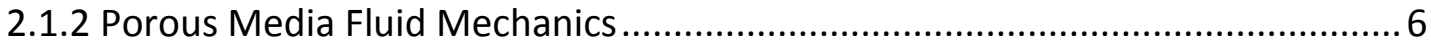

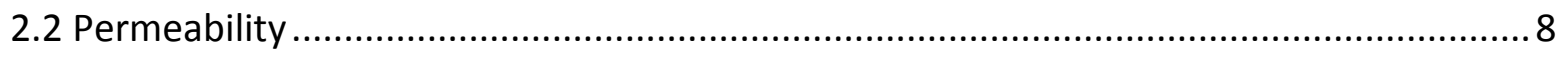

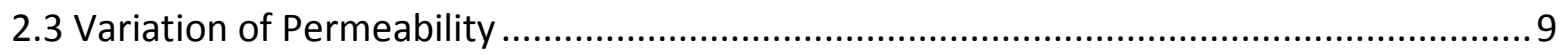

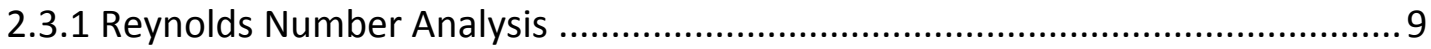

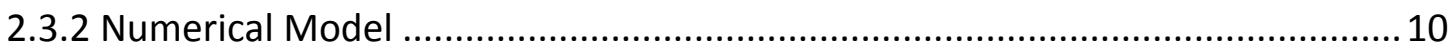

2.3.3 Results of Permeability Variation Study ................................................... 16

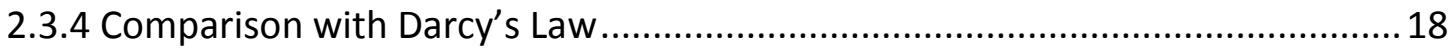

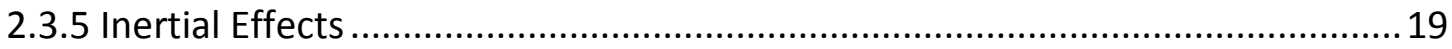

2.3.6 Conclusions from Permeability Variation Study ........................................ 19

3 Model Development $\quad 20$

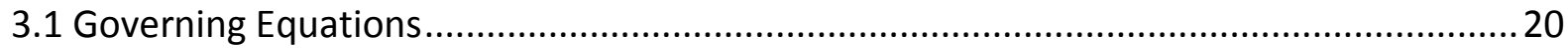

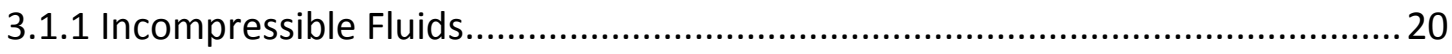

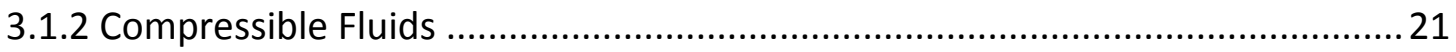

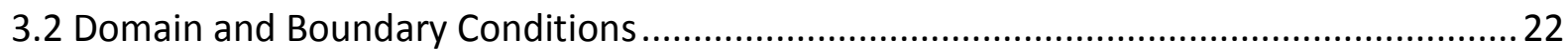




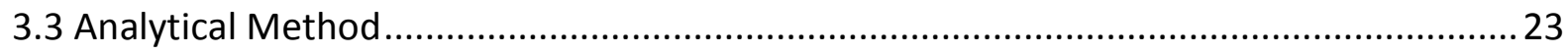

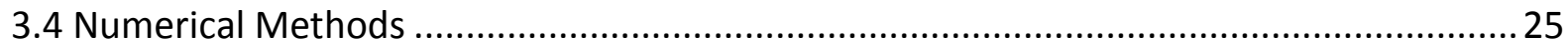

3.4.1 Discretization of the Governing Equations - Incompressible..........................25

3.4.2 Discretization of the Governing Equations - Compressible ............................. 26

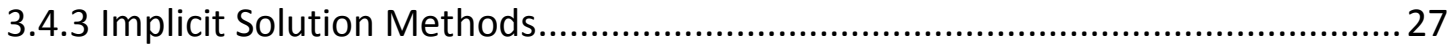

3.4.4 Explicit Solution Method - Incompressible Flow........................................ 28

3.4.5 Explicit Solution Method - Compressible Flow ......................................... 30

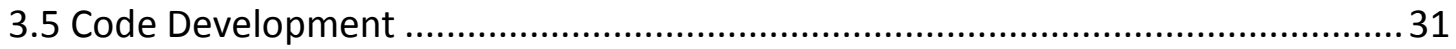

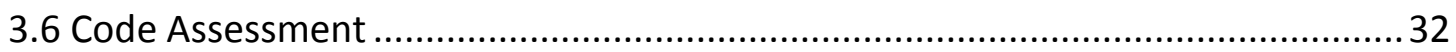

$\begin{array}{ll}4 \text { Results and Discussion } & 34\end{array}$

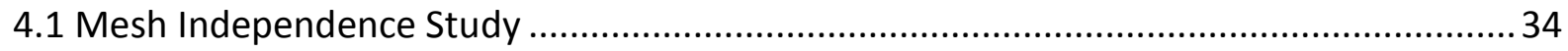

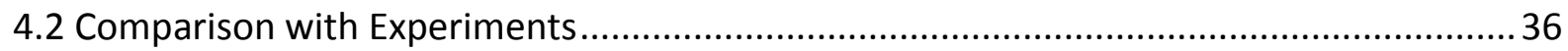

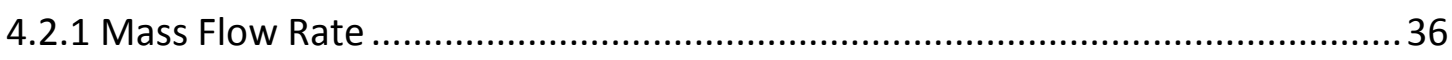

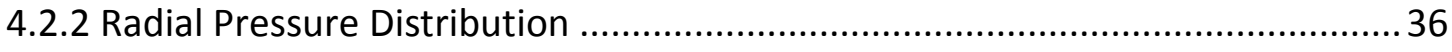

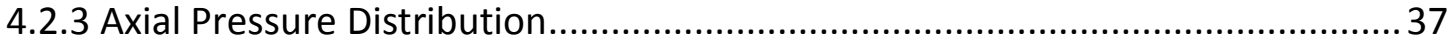

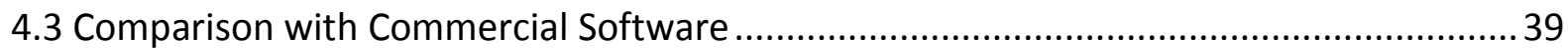

5 Conclusions and Future Work $\quad 42$ 
Nomenclature

\section{Subscripts}

$x$

y

Z

f

p

total

u

d

0

I

$\mathrm{fp}$

bp

e

V

\section{Constants}

R

air ideal gas constant $=386.8\left(\frac{\mathrm{J}}{\mathrm{kg} * \mathrm{~K}}\right)$

$\mathrm{D}_{0}$ center difference operator 


\section{Variables}

F

K

k

C

C

M

$\mathrm{p}$

$\mu$

K

v

$\mathrm{K}_{1}$

$\rho$

a

B

$\varepsilon$

$a_{v}$

d

$\operatorname{Re}$

$\dot{m}$

$D_{s}$

$D_{b}$

$\mathrm{N}$

$\phi$ seal force vector $(\mathrm{N})$

seal direct stiffness

seal cross-coupled stiffness

seal direct damping

seal cross-coupled damping

seal mass coefficient

pressure

viscosity

permeability

velocity vector

inertial permeability

fluid density

inertial empirical resistance coefficient

viscous empirical resistance coefficient

porosity

area to volume ratio

bristle diameter

porous media flow path length

Reynolds number

mass flow rate

overall seal outside diameter

bristle bore diameter

number of bristles per unit length of circumference

bristle lay angle 


$\begin{array}{ll}P^{*} & \begin{array}{l}\text { nondimensional pressure } \\ Z\end{array} \\ R_{p} & \text { axial pressure tap location } \\ t & \text { time } \\ R_{0} & \text { outer radius } \\ R_{i} & \text { inner radius } \\ Y & \text { diagonal coefficient of numerical scheme } \\ \alpha_{1} & \text { superdiagonal coefficient of numerical scheme } \\ \alpha_{2} & \text { subdiagonal coefficient of numerical scheme } \\ \beta_{1} & \text { forward column coefficient of numerical scheme } \\ \beta_{2} & \text { backward column coefficient of numerical scheme } \\ T & \text { temperature (K) } \\ {[A]} & \text { total scheme coefficient matrix } \\ \{p\} & \text { total grid pressure vector } \\ \{\mathrm{b}\} & \text { total boundary condition vector } \\ {[D]} & \text { diagonal matrix } \\ {[R]} & \text { remainder matrix }\end{array}$




\section{Chapter 1}

\section{Introduction}

\subsection{Overview}

Brush seals are a proven technology used in turbomachinery for the purpose of reducing leakage and controlling flow. Some applications of brush seals include interstage sealing and end sealing of turbines (Pastrana, Wolfe, Turnquist, \& ME, 2001) as well as compressor discharge seals (Hendricks, et al., 1994). A typical brush seal is designed to be fixed to the stator with compliant bristles extending toward the rotor (Fig. 1.1). The bristles are partially covered upstream by a front face plate and downstream by a back plate. Individual designs often differ in bristle material, bristle packing density, clearance/interference, front and backing plate heights, bristle lay angle, and pressure balancing methods (Pekris, Franceschini, \& Gillespie, 2012; Li \& Yang, Analytical Investigations on the Contact Force Between Bristle Packs and Shaft Surface of Brush Seals, 2012; Fellenstein \& DellaCorte, 1995).

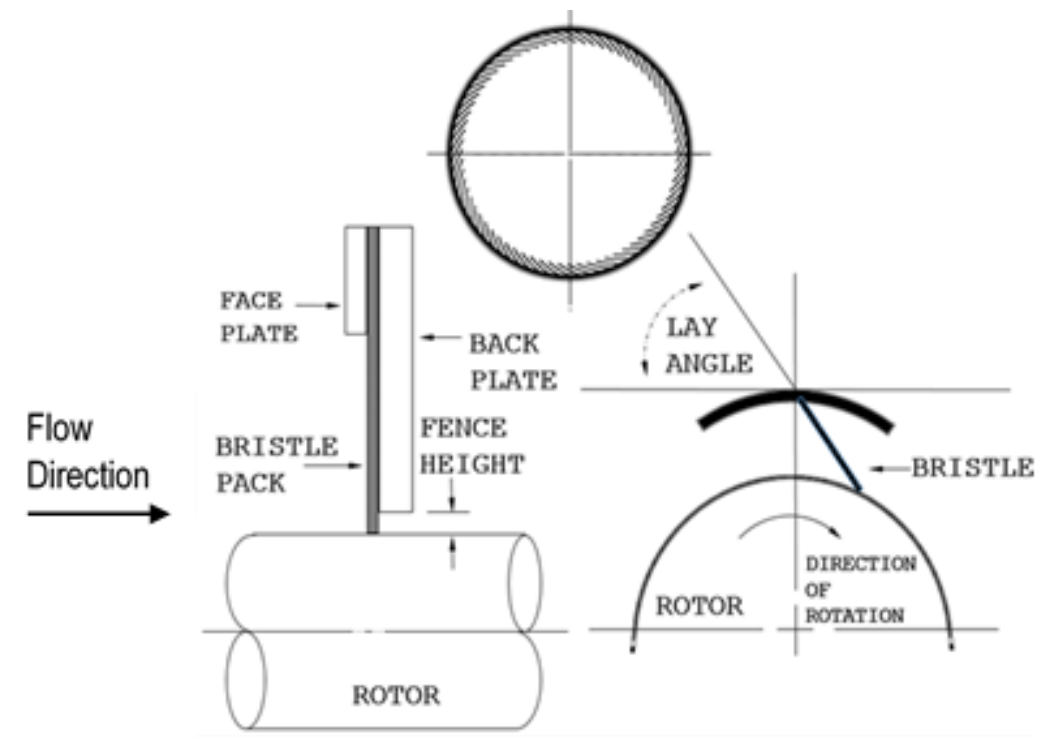

Figure 1.1 Common brush seal features and design characteristics. The bristles are oriented in the direction of rotation 
The earliest use of brush seals began in the 1950s when they were implemented for inter-stage compression sections of jet engines (Cieślewicz, 2004). They became prevalent in jet engines during the late 1980s and early 1990s (Pastrana, Wolfe, Turnquist, \& ME, 2001). Today they are commonly applied to many different turbomachines.

The primary function of any annular seal is to reduce the leakage flow between rotating and stationary parts. In order to asses and predict a seal's ability to accomplish this task, many different methods are used. Experimental testing of brush seals has resulted in an improvement of the understanding of the internal fluid mechanics. Flow visualization tests have confirmed that the flow is highly chaotic and many vortices are formed due to the "flow over cylinder" around the bristles (Braun, Hendricks, \& Canacci, 1990). CFD studies have also been an effective modeling tool in the design and analysis of brush seals.

\subsection{Methods of Analysis}

There are two standard methods of predicting the leakage through a brush seal: Bulk flow analysis and computational fluid dynamics (CFD). Both of these methods typically rely on a porous media model to calculate flow properties through the bristle pack. The focus of this project was to develop a new model for predicting the performance of a standard brush seal, and to solve the resulting system of equations by employing a finite difference method.

\subsubsection{Computational Fluid Dynamics}

The use of CFD modeling is common for most annular seals. The process requires a domain to be specified, divided into smaller sub-domains, and then discretized into a grid. Governing equations are then solved using numerical approximations throughout the grid. Environmental parameters such as inlet pressure and shaft rotation are expressed as boundary conditions, and flow parameters are calculated at each node. This method is very useful for situations that require very detailed information of the solution, or when a great deal of parameter variation must be considered, such as a geometric optimization study. The drawback of CFD analysis is that it requires a large amount of time and effort. A simulation for a grid that consists of several million elements can take several hours or days, even on a sophisticated computer. Model validation and mesh-independence is required for most situations in order to establish that the results are reliable. This can add a great deal of time to the analysis process and requires the user to be knowledgeable on the subject of mesh generation. 


\subsubsection{Bulk Flow Method}

Bulk flow methods are employed to predict leakage and rotordynamic properties for many different types of annular seals (Nordmann \& Weiser, 1991; Williams \& Flack, 1998; Migliorini, Untaroiu, \& Allaire, 2012). This method involves dividing the seal domain into a few control volumes (typically less than four) and solving simplified versions of the momentum, continuity, and energy equations. A few critical assumptions are made about the shear stress distribution in the fluid, and the turbulent behavior within the control volumes.

\subsection{Prior Work}

The desire to improve the design of brush seals necessitates a further understanding of the fluid dynamics within an operating brush seal. Due to the bristles, high pressure gradients, and the rotating shaft the flow situations can be very complex and chaotic. This makes it very difficult to model a brush seal and predict fluid forces by using strictly analytical methods. Most of the techniques that have been employed to study brush seals involve simplifying assumptions about the properties of the fluid or the boundary conditions. An example of an analytical study can be found in (Saber \& Abdou, 2010) where a strip seal is modeled by using the Navier-Stokes and continuity equations and assuming a steady, laminar, incompressible and iso-viscous flow. A strip seal is similar to a brush seal, but implements strips instead of bristles. The flow was modeled by a system of non-dimensionalized PDE's, and the solutions were helpful for demonstrating qualitative information about how certain design parameters affect the performance of the seal. From this analysis conclusions were drawn on the basic effects of strip angle and spacing, the sensitivity to strip thickness, and the effect of clearance between strip and shaft. This type of information can be extremely valuable for engineers who seek to understand the basic principles of a seal, but in order to find out specific values of physical properties within an operating seal we will need a different method of analysis.

Bristle Modeling: The forces acting on the rotor due to rotor-bristle interaction can be calculated using the finite element method. The study of these forces is a very difficult threedimensional problem. During operation the bristles will deflect radially due to interaction with the rotor, and they will deflect axially due to pressure loading. A study conducted by Aksit (Aksit, 2012) used a FE method with the bristles modeled as 3D beam elements. From this analysis it was shown that the bristle tip forces are primarily determined by the pressure loading. Another publication by Qiu and Li describes the use of finite element and CFD software together in order to calculate bristle deflection and frictional heating (Qiu \& Li, Numerical Investigations on the Heat Transfer Behavior of Brush Seals Using Combined Computational Fluid Dynamics and Finite Element Method, 2013). The bristle pack was modeled in a CFD software package as a porous medium, and the bristles were modeled in a FE software package as beam elements. An iterative 
method was used to calculate pressure and contact forces. The CFD simulation was used to obtain the pressure loading on the bristle pack, and the FE method was then used to determine the bristle deflection. The result was then used to modify the CFD model and calculate new values.

Experimental Techniques: Some previous work has examined the nature of the flow within the seal to determine details of the velocity profile, turbulent eddies, and pressure distribution. It is commonly accepted that the flow within the seal, particularly within the bristle pack is chaotic and varies greatly with the application, fluid type, operating conditions, and seal design. A paper by Braun, Hendricks, and Canacci (Braun, Hendricks, \& Canacci, 1990) describes a flow visualization technique that was used to provide insight. A linear seal configuration was tested in both an oil tunnel and a water tunnel. The test section of the tunnel was supplied with working fluid by gravity, the volumetric flow rate was maintained constant, and the fluid was seeded with light-reflecting particles of magnesium oxide. A laser was mounted above the test section and used to shine through the fluid in order to make the particles visible. The dynamics of the fluid and bristles were recorded on video tape, and provide a qualitative demonstration of the internal behavior of the seal. Pressure taps along the axis of the seal provided a map of pressure drop in the flow direction.

The effects of the lay angle on the seal performance were considered in the research of Crudginton, Bowsher, and Lloyd (Crudgington, Bowsher, \& Lloyd, 2009). This study took a comprehensive approach by using analytical, experimental, and numerical techniques. The influence of lay angle on the bristle tip pressure (BTP) was first modeled using simple beam theory. Estimates of the bristle tip pressure were calculated and discussed. Experiments were conducted using two brush seals with different lay angles. The two angles were approximately $47^{\circ}$ and $41^{\circ}$. The tests showed that measured BTP was greater than calculated, which can be expected because the friction between adjacent bristles is ignored in the simple beam theory calculations. The BTP was higher for the smaller lay angle. Among the conclusions of the study were: Contact pressure increases more with pressure drop when the lay angle is greater, and blow down effect is more pronounced at higher lay angles.

Bulk Flow Techniques: The first bulk flow model for brush seals was created by Hendricks and Schlumberger (Hendricks \& Schlumberger, 1991). The bulk flow code was capable of predicting mass flow for seals with different geometric parameters and also took bristle loadings into account. There have been several updates to this code and its ability to predict seal performance. 


\section{Chapter 2 \\ Seal Performance Modeling}

\subsection{Theoretical Background}

A complete study of an annular seal for turbomachinery applications should address leakage performance as well as the seals influence on rotordynamic stability. Shaft vibration must be kept below a certain threshold limit to ensure safe and reliable operation. Much of the shaft vibration can be attributed to imbalance or operation at a critical speed, but some components of the vibration can also be the result of aerodynamic and fluid-film cross-coupling forces generated in the seals and bearings (Yoon, 2013). In this chapter, a brief discussion of the rotordynamic performance of brush seals is given, followed by a more in-depth discussion of the fluid mechanics.

\subsubsection{Brush Seal Rotordynamics}

Brush seals are primarily used for scenarios where the fluid to be sealed is a gas. The forces acting on the rotor at any particular cross-section are considered to be functions of the shaft motion within the plane:

$$
-\left\{\begin{array}{l}
F_{x} \\
F_{y}
\end{array}\right\}=\left[\begin{array}{cc}
K & k \\
-k & K
\end{array}\right]\left\{\begin{array}{l}
X \\
Y
\end{array}\right\}+\left[\begin{array}{cc}
C & c \\
-c & C
\end{array}\right]\left\{\begin{array}{c}
\dot{X} \\
\dot{Y}
\end{array}\right\}+M\left\{\begin{array}{l}
\ddot{X} \\
\ddot{Y}
\end{array}\right\}
$$




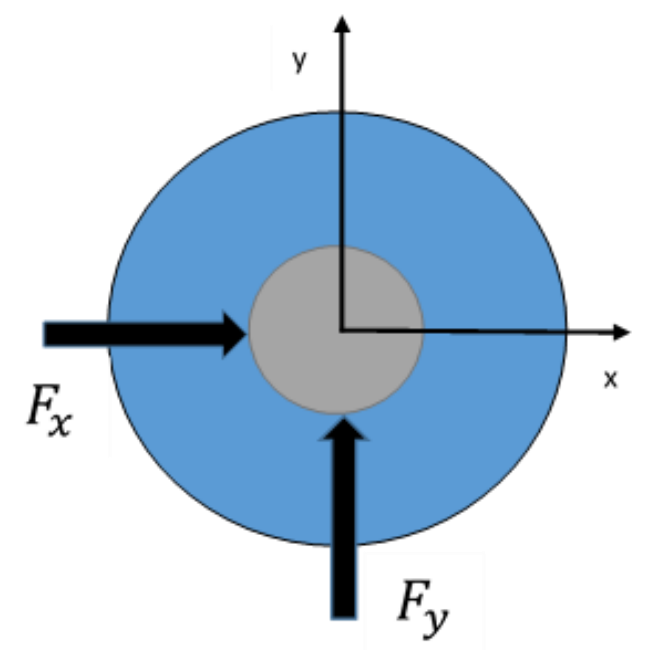

Figure 2.1 Net stiffness and damping forces acting on rotor

Where $[K]$ is the seal stiffness matrix, $[C]$ is the seal damping matrix and $M$ is the mass coefficient. For seals gas seals such as a brush seal, the added mass term is typically considered to be negligible and the governing equation is reduced to:

$$
-\left\{\begin{array}{c}
F_{x} \\
F_{y}
\end{array}\right\}=\left[\begin{array}{cc}
K & k \\
-k & K
\end{array}\right]\left\{\begin{array}{l}
X \\
Y
\end{array}\right\}+\left[\begin{array}{cc}
C & c \\
-c & C
\end{array}\right]\left\{\begin{array}{l}
\dot{X} \\
\dot{Y}
\end{array}\right\}
$$

For further information on the derivation of these equations the reader is referred to Childs' textbook (Childs, 1993).

The off-diagonal terms of the stiffness and damping matrices are referred to as crosscoupled stiffness $k$, and cross-coupled damping $c$, respectively. These terms of are great interest to rotordynamicists because they are primarily responsible for the stability of the rotor-seal system. From equation 2.1.2 it can be seen that the rotordynamic forces are functions of eccentric shaft motion. For annular seals the coefficients in equation 2.1.2 depend on several factors including the pressure distribution within the seal. They are typically determined experimentally (Aksit, 2012), but may also be approximated by analytical and numerical methods (Vance, 2010).

The work presented in this thesis is limited to the study of brush seals in situations where the shaft is not rotating. As a result, the estimation of rotordynamic coefficients is compared to static testing.

\subsubsection{Porous Media Fluid Mechanics}

The majority of brush seal CFD models that are presented in the literature use a porous media approach to model the bristle pack. The basic theory is to treat the region as a continuum that provides a resistance to flow. The basic constitutive equation describing flow 
through porous media is Darcy's law, which relates flux, fluid viscosity, and pressure gradient by using a resistance coefficient known as permeability $(\mathrm{k})$ :

$$
\nabla p=-\frac{\mu}{\boldsymbol{\kappa}} \boldsymbol{v}_{f}
$$

Darcy's law, which is an expression of conservation of momentum, was first determined experimentally. However, it has also been shown that it can be derived from the Navier-Stokes equations. An extension of Darcy's law is known as the Darcy-Forchheimer Law. This is a more complete model of flow through porous media at higher Reynolds numbers as it accounts for inertial effects (Jambhekar, 2011). The Darcy-Forchheimer law can be expressed in equation form as:

$$
\nabla p=-\frac{\mu}{\boldsymbol{\kappa}} \boldsymbol{v}_{f}-\frac{\rho}{\boldsymbol{\kappa}_{\mathbf{1}}}\left|\boldsymbol{v}_{f}\right| \boldsymbol{v}_{f}
$$

In the most general case, the coefficients $\mathrm{k}$ and $\mathrm{K}_{1}$ are second order tensors with nine components. They can be written as $\mathrm{K}_{\mathrm{ij}}$ and $\mathrm{k}_{1 \mathrm{ij}}$. However, off-diagonal terms are typically neglected in the analysis of brush seals $\mathrm{K}_{\mathrm{ij}}=\mathrm{k}_{1 \mathrm{ij}}=0$ for $\mathrm{i} \neq \mathrm{j}$. The Forchheimer model was applied to the study of a brush seal by Dogu (Dogu, 2005). The coefficients of velocity were written more simply to include density and viscosity:

$$
-\frac{d p}{d x_{i}}=\left(a_{i j}\left|u_{j}\right|+B_{i j}\right) u_{j}
$$

Eq. 2.1.5 is an alternate form of Eq. 2.1.4, where index notation is used instead of tensor notation. The two permeability coefficients, a and B, were defined for each spatial direction, resulting in a total of 6 coefficients. These coefficients were determined empirically by collecting leakage data, the axial pressure distribution on the rotor surface, and the radial pressure distribution on the backing plate. A CFD model of a brush seal was created based on experimental data. This study contained detailed information on the seal dimensions and the boundary conditions, which made it possible to compare the CFD results against the experimental results. The CFD study was performed in two dimensions (radial and axial) because the circumferential velocity was negligibly low. The calibrated permeability coefficients are listed in Table 2.1. 
Table 2.1. Resistance coefficients reported by Dogu [9].

\begin{tabular}{c|c|c|}
\cline { 2 - 3 } radial direction & a (Inertial) & $\mathrm{B}$ (Viscous) \\
\cline { 2 - 3 } axial direction & $1 \times 10^{5}$ & $1 \times 10^{5}$ \\
\cline { 2 - 3 } & $7.5 \times 10^{6}$ & $4.5 \times 10^{7}$ \\
\cline { 2 - 3 } & {$\left[\mathrm{kg} / \mathrm{m}^{4}\right]$} & {$\left[\mathrm{kg} / \mathrm{s} \cdot \mathrm{m}^{3}\right]$}
\end{tabular}

The results from this study demonstrated that a bulk porous medium approach with calibrated permeability coefficients can be used to model the flow through a brush seal with a high degree of accuracy. This method provides an effective tool to study and predict leakage, but it is limited in its ability to provide information about the rotordynamics of the system. From a CFD analysis in a software package such as ANSYS CFX, flow field and pressure field data could be used to obtain pressure forces, which could then be used for rotordynamic stability analysis. When modeling a bristle pack as a porous medium in CFD, one of the major tasks is accurately calibrating the resistance coefficients. If the model is to be relied upon for future calculations it must first be validated against experimental data for leakage through the seal for a given pressure distribution. The resistance coefficients can then be adjusted to obtain agreement between the simulation and the experiment. In an experiment conducted by Pugachev and Helm (Pgachev \& Helm, 2008), a series arrangement of seals was tested and then used to calibrate a CFD model of the arrangement. In this study they succeeded in calibrating the porous model to give good agreement between the simulations and the experiments.

\subsection{Permeability}

The permeability in Darcy's law is the defining material property that may be used to characterize the tendency of a porous medium to pass a fluid. In the context of an annular seal, with all external conditions being equal, the permeability will determine the leakage rate. It is important to note that the proportionality constants in Eq. 2.1.5 are sometimes loosely referred to as permeability. For this paper, permeability will refer to $k$ and $\kappa_{1}$, which are the intrinsic properties of the medium, and do not depend on the fluid properties.

As mentioned above, a general permeability tensor is of rank two and has nine components which are expressed in matrix form as:

$$
\boldsymbol{\kappa}=\left[\begin{array}{lll}
\kappa_{11} & \kappa_{12} & \kappa_{13} \\
\kappa_{21} & \kappa_{22} & \kappa_{23} \\
\kappa_{31} & \kappa_{32} & \kappa_{33}
\end{array}\right]
$$

Previous work has demonstrated the utility of the isotropic porous medium model of a brush seal for prediction of leakage [11]. This assumption implies that off-diagonal elements are zero, and that all diagonal elements have the same value. Therefore the permeability and inertial 
permeability tensors reduce to scalars $\mathrm{K}$ and $\mathrm{K}_{1}$, respectively. In a study by Chew and Hogg (Chew \& Hogg, Porosity Modeling of Brush Seals, 1997) the isotropic assumption was applied to a brush seal model. Results from this model were observed to be in good agreement with empirical tests from multiple sources (Bayley \& Long, 1993; Carlile, Hendricks, \& Yoder, 1993; O'Neil, 1993).

Although the simplifying assumption of constant permeability can be useful in some situations, it seems intuitive that effects such as bristle flutter, blow down, and bending could cause significant variation in the permeability of the bristle pack. Many previous studies have addressed the tendency of resistance coefficients to vary. The relationship between the resistance coefficients and the pressure drop can be modeled by the Ergun equation (Ergun, 1952):

$$
\frac{\partial p}{\partial x}=\frac{150 \mu(1-\varepsilon)^{2}}{D_{p}^{2} \varepsilon^{3}} u+\frac{1.75 \rho(1-\varepsilon)}{D_{p} \varepsilon^{3}} u^{2}
$$

Where: $p=$ pressure, $\varepsilon=$ porosity, and $u=$ fluid velocity in $\mathrm{x}$ direction. This model has been used extensively in the literature (Li, Experimental and Numerical Investigations on the Leakage Flow Characteristics of the Labyrinth Brush Seal, 2012; Qiu, Li, \& Yan, Investigation Into the Flow Behavior of Multi-Stage Brush Seals, 2014). The parameter $D_{p}$ is referred to as "equivalent mean particle diameter". The name for this parameter comes from its original application to flow through packed beds (Ergun, 1952). $D_{p}$ is calculated from the bristle geometry:

$$
a_{v}=\frac{S_{\text {total }}}{V_{\text {total }}}=\frac{\pi d l}{\left(\frac{\pi d^{2}}{4}\right) l}=\frac{4}{d}, D_{p}=\frac{6}{a_{v}}=1.5 d
$$

Where: $a_{v}=$ area to volume ratio, $S_{\text {total }}=$ total bristle surface area, $V_{\text {total }}=$ total bristle volume, $l=$ axial length, and $d=$ bristle diameter.

In (Dogu, 2005) Dogu asserts that bristle pack thickness will decrease only until the bristles are compressed together and against the back plate. After this point is reached, the porosity, pack thickness, and resistance coefficients will all remain constant. This of course requires that the pressure difference is sufficiently high for that particular seal.

\subsection{Variation of Permeability}

A study was conducted to demonstrate the change in permeability of a brush seal as a function of pressure drop using CFD. This work utilized experimental data that is reported by Bayley and Long in (Bayley \& Long, 1993). In their study the ratio of inlet to outlet pressure was varied, and mass flow was measured for several different pressure ratios. First, this set of data was analyzed in a new way to establish further understanding of the fluid mechanics. From this analysis a CFD model was created and iteratively calibrated to find the permeability of the bristle pack at every value of pressure ratio. This provided a mapping of how the permeability changed 
over the range of pressure ratios. The results of this study provided a new and simplified basis for the study of flow in a brush seal and for using a porous media model of the bristle pack.

\subsubsection{Reynolds Number Analysis}

Although Eq. 2.1.4 is the more general model for flow through porous media, Eq. 2.1.3 has been shown to be sufficient for many applications where the viscous forces have a significant influence. The Reynolds number may be calculated as:

$$
\operatorname{Re}=\frac{\rho D_{p} \mathrm{u}}{\mu}
$$

By reformulating the expression in terms of mass flow rate we obtain:

$$
R e \approx \frac{\rho \mathrm{D}_{\mathrm{p}}}{\mu}\left(\frac{\dot{m}}{\rho A}\right)=\frac{\dot{m} D_{p}}{A \mu}
$$

One of the standards for determining when the flow regime should be modeled by Eq. 2.1.3 or Eq. 2.1.4 was developed by Chilton and Colburn in (Chilton \& Colburn, 1931). The critical region for non-Darcian effects to become significant was found to be when the Reynolds number was in the range $40-80$. In order to apply Eq. 2.3.2 to a brush seal model, the area $A$ can be approximated as an annulus defined by the bristle pack. This results in a final equation for the Reynolds number:

$$
R e \approx \frac{\dot{m} D_{p}}{\mu \pi\left[\left(\frac{D_{s}}{2}\right)^{2}-\left(\frac{D_{b}}{2}\right)^{2}\right]}
$$

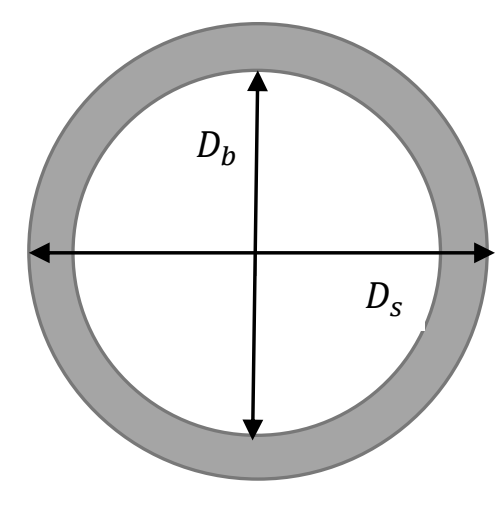

Figure 2.3.1 Annular cross section of the seal outlet (axial view)

where $D_{s}$ is the overall outside diameter of the seal and $D_{b}$ is the bore diameter. This equation, along with the criteria from Chilton and Colburn (Chilton \& Colburn, 1931), was used as the basis 
for determining whether to model the flow with Eq. 2.1.3 or Eq. 2.1.4, as described in a later section.

\subsubsection{Numerical Model}

A CFD model was developed based on the given dimensions and properties of the seal described in (Bayley \& Long, 1993). Although the seal domain being studied has rotational symmetry about the axis of the shaft, and could therefore be modeled with a two-dimensional domain, the CFD model was three dimensional. The purpose for this was to maintain generality and to ensure that no unexpected three-dimensional effects (such as turbulent eddies) were being eliminated preemptively. A 10-degree sector model was constructed in ANSYS CFX 15 (Fig. 2.3.2). Due to the circumferential symmetry it is assumed that a sector model of this size is sufficient to model the entire seal (Untaroiu, Liu, Migliorini, Wood, \& Untaroiu, 2014). The experimental testing was performed with a nonrotating shaft, therefore shaft rotation was not considered in this study. A periodic boundary condition was used to connect the two sides of the sector model. The porosity of the bristle pack was estimated by the method described by Chew and Hogg in (Chew \& Hogg, Porosity Modeling of Brush Seals, 1997).

$$
\varepsilon=1-\frac{\pi d^{2} N}{4 l \sin \phi}
$$

For the seal described in (Bayley \& Long, 1993) the values for these parameters are: $d=0.0762$ $\mathrm{mm}, \mathrm{N}=2400$ bristles/in, I $=0.7 \mathrm{~mm}, \varphi=45^{\circ}$. Therefore, the value of porosity is estimated to be $\sim 0.129$. However, this value is expected to vary to some extent as pressure ratio changes, so a sensitivity study was performed for the CFD model. It was found that simulation results do not change for a range of porosity between 0.11 and 0.35 . The seal inlet and outlet were defined as openings with pressure boundary conditions. The outlet pressure was fixed at $100 \mathrm{kPa}$ for every simulation, while the inlet pressure was varied between $107 \mathrm{kPa}$ and $380 \mathrm{kPa}$. The rotor and stator were defined as smooth, no-slip walls.

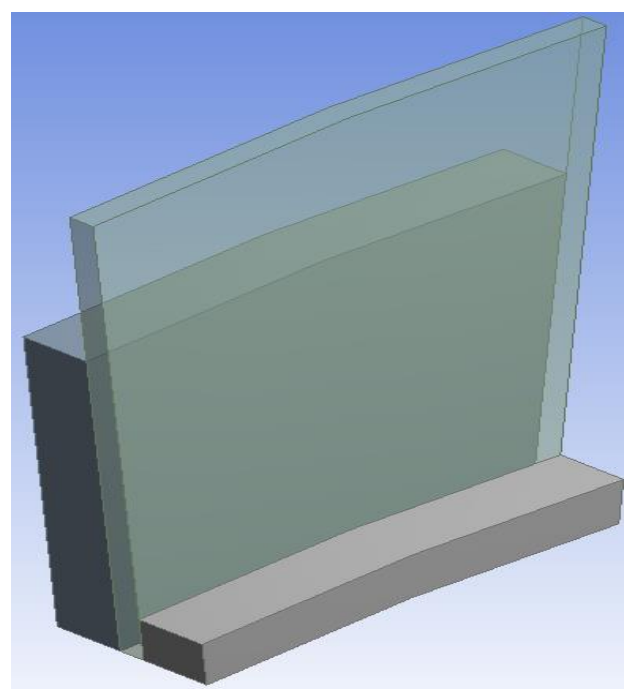

Figure 2.3.2. Geometry of a sector of the brush seal, including the back plate, bristle pack, and face plate. 
There is no information available on the regions upstream or downstream of the seal that was tested in (Bayley \& Long, 1993). Because this particular seal was single-stage, it can be assumed that the regions before and after the seal are defined by the annular space between the rotor and the stator. The influence of upstream and downstream regions was tested by creating a computational model that includes extended inlet and outlet regions (Fig. 2.3.3).

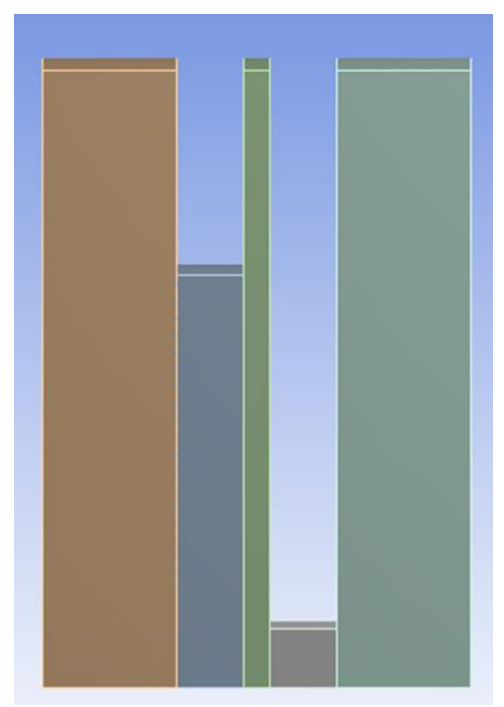

Figure 2.3.3. Geometry of a sector of the brush seal, including upstream and downstream regions.

Flow rate calculations using the model with extended inlet and outlet regions were compared to calculations for the same operating conditions without the extended regions. The operating conditions tested were those corresponding to the lowest, middle, and highest values of pressure ratio. The results from the simulations using the extended model were within $0.1 \%$ agreement with the results from the non-extended model (Fig. 2.3.4). This agreement in simulation results is used as justification to conduct the rest of the study with a smaller computational domain. The smaller model begins at the front plate of the seal and ends at the back plate.

Studying the change in permeability at several operating pressures required many CFD simulations to be run for each operating point. This made it necessary to choose a mesh capable of providing enough detail of the flow field to ensure accuracy while minimizing computational time. Mesh independence was determined by testing 11 different meshes ranging in size from 5,684 to $6,000,000$ elements (Fig. 2.3.5). It was found that the CFD simulation results became independent of mesh size at approximately 79,000 elements, and the $2 \times 10^{6}$ element mesh that was used for this study was decidedly in the range of mesh independence (Fig. 2.3.6). 


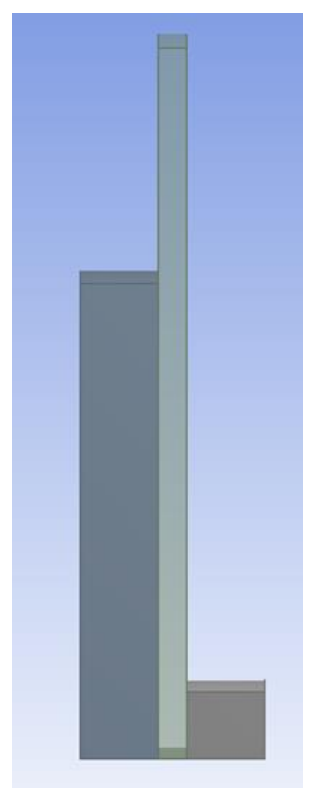

Figure 2.3.4. Geometry of a sector of the brush seal with no upstream or downstream regions.

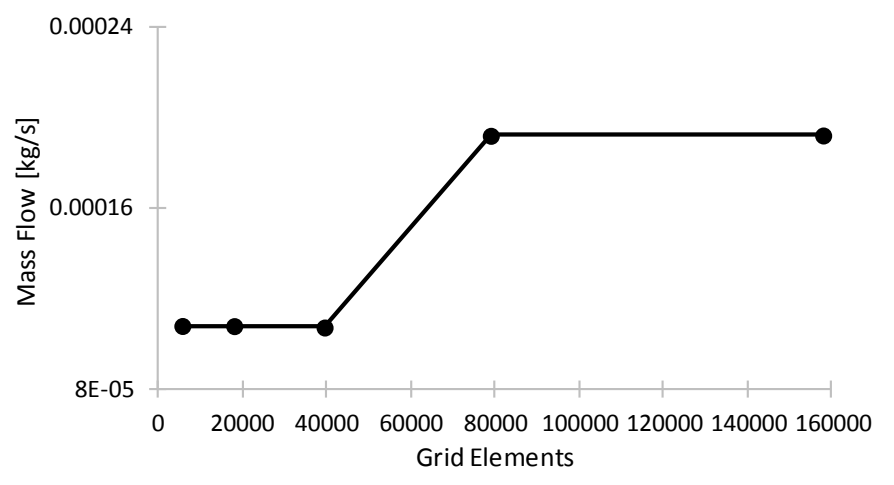

Figure 2.3.5. Mesh independence study.

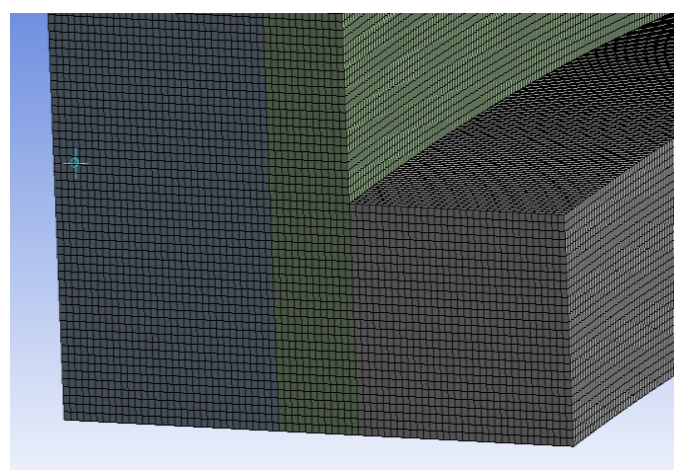

Figure 2.3.6. Mesh applied to the analysis domain. 
The Shear Stress Transport model was chosen to model turbulence and the temperature conditions were treated as isothermal with an air inlet temperature of $20^{\circ} \mathrm{C}$. The convergence criteria specified were RMS residuals below $10^{-4}$ to minimize any sources of numerical error.

Because this CFD model is based off of the seal tested in (Bayley \& Long, 1993), it was important to compare the model to the available experimental data. Axial and radial pressure distributions were measured and reported in terms of a nondimensional pressure $\mathrm{P}^{*}$. The value of nondimensional pressure is calculated by the following equation:

$$
P^{*}=\frac{p-p_{d}}{p_{u}-p_{d}}
$$

The radial pressure distribution was originally measured by five different pressure taps on the back plate of the seal. The axial pressure distribution was measured by 24 pressure taps in the rotor, 11 of which were located under the bristle tips. The coordinate system for the radial direction was defined by $Y$, which is the ratio of the radial distance from the rotor divided by the distance between the shaft and the bottom of the front plate. For the axial pressure distribution, the location of the pressure tap can be determined from $Z$, the tap number. The bristle pack starts at $Z=5$ and ends at $Z=16$. Bayley and Long presented pressure distribution data by focusing on specific ranges of pressure ratio, and then averaging the pressure distribution from all the tests conducted in the range. For the sake of comparison, Figures 2.3.7 and 2.3.8 show the pressure distributions from the CFD simulations along with the measured pressure distributions, showing reasonably good agreement between experimentally and numerically values.

Experimental data for mass flow rate as a function of pressure ratio was obtained from (Bayley \& Long, 1993) and is listed in Table 2.2. Due to the relatively even spacing of the data points, it was determined that using every other set of points would be sufficient for characterizing the change in permeability.

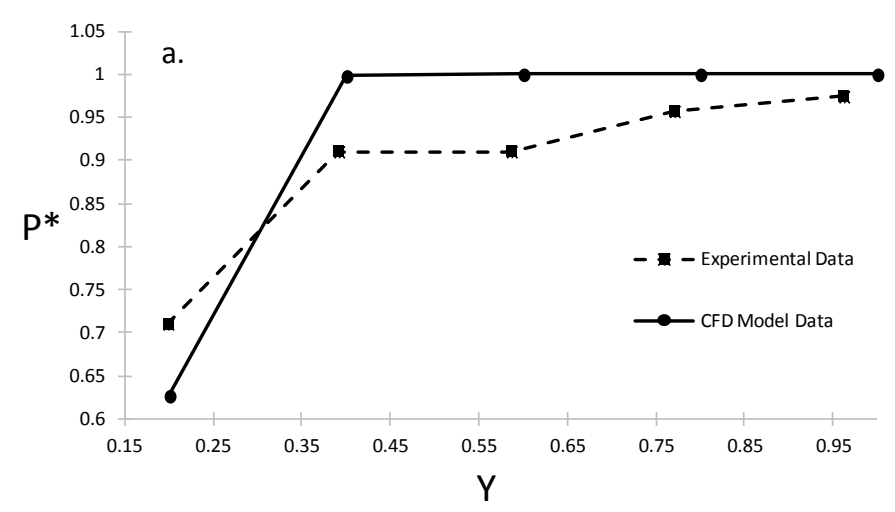




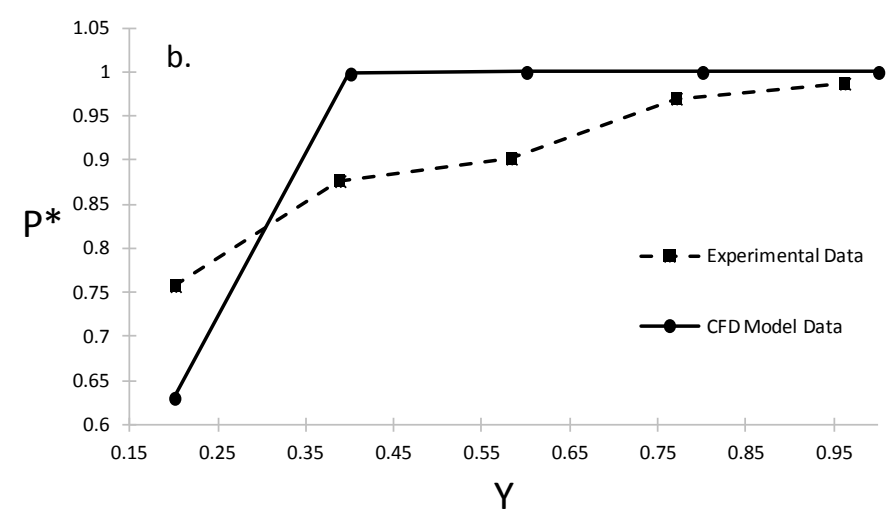

Figure 2.3.7. Comparisons of radial pressure distributions between the CFD model and experimental data from [12] for a. pressure ratios of 1.29-1.86 and b. ratios of 3.17-3.80.

The Reynolds numbers listed in Table 2.3.1 were found by applying Eq.2.3.2 to each data point, showing that the Reynolds number for each point was well below the range for non-Darcian effects to be significant. Because of this, Eq. 2.1.3 was found to be sufficient to simulate these experiments using CFD.

\begin{tabular}{|c|c|c|}
\hline Pressure Ratio & Flow Rate (kg/s) & Reynolds Number \\
\hline 1.19 & $1.86 \mathrm{E}-03$ & 1.82 \\
\hline 1.32 & $2.08 \mathrm{E}-03$ & 2.03 \\
\hline 1.36 & $3.04 \mathrm{E}-03$ & 2.96 \\
\hline 1.89 & $4.81 \mathrm{E}-03$ & 4.70 \\
\hline 2.12 & $5.82 \mathrm{E}-03$ & 5.68 \\
\hline 2.34 & $5.82 \mathrm{E}-03$ & 5.68 \\
\hline 2.47 & $7.03 \mathrm{E}-03$ & 6.87 \\
\hline 2.64 & $7.03 \mathrm{E}-03$ & 6.87 \\
\hline 2.69 & $8.73 \mathrm{E}-03$ & 8.53 \\
\hline 2.92 & $8.73 \mathrm{E}-03$ & 8.53 \\
\hline 3.14 & $1.06 \mathrm{E}-02$ & 10.31 \\
\hline 3.40 & $1.28 \mathrm{E}-02$ & 12.46 \\
\hline 3.70 & $1.35 \mathrm{E}-02$ & 13.15 \\
\hline 3.99 & $1.28 \mathrm{E}-02$ & 12.46 \\
\hline
\end{tabular}

Table 2.3.1 Experimental data of brush seal leakage.

For this study, an isotropic model for the bristle pack was used due to the lack of non-Darcian effects expected. Because the shaft was not rotating during their experiment, no rotor excursions were present and the circumferential velocity of the fluid was minimal. The expected result is that changes in permeability would occur predominantly in the axial direction due to the large pressure difference between the axial ends of the seal. However, in a rotating environment it is noted that sharp lay angles and rotational flow could significantly contribute to variations in permeability in the radial direction as well. 
For a given operating pressure, several CFX input files were generated with all conditions identical except for the permeability. An initial "guess" value was chosen to be in the middle of the range of permeability used. The range of values considered for matching the model predictions to the experimental data were based on experimental values previously reported in the literature for brush seals (Dogu, 2005). For
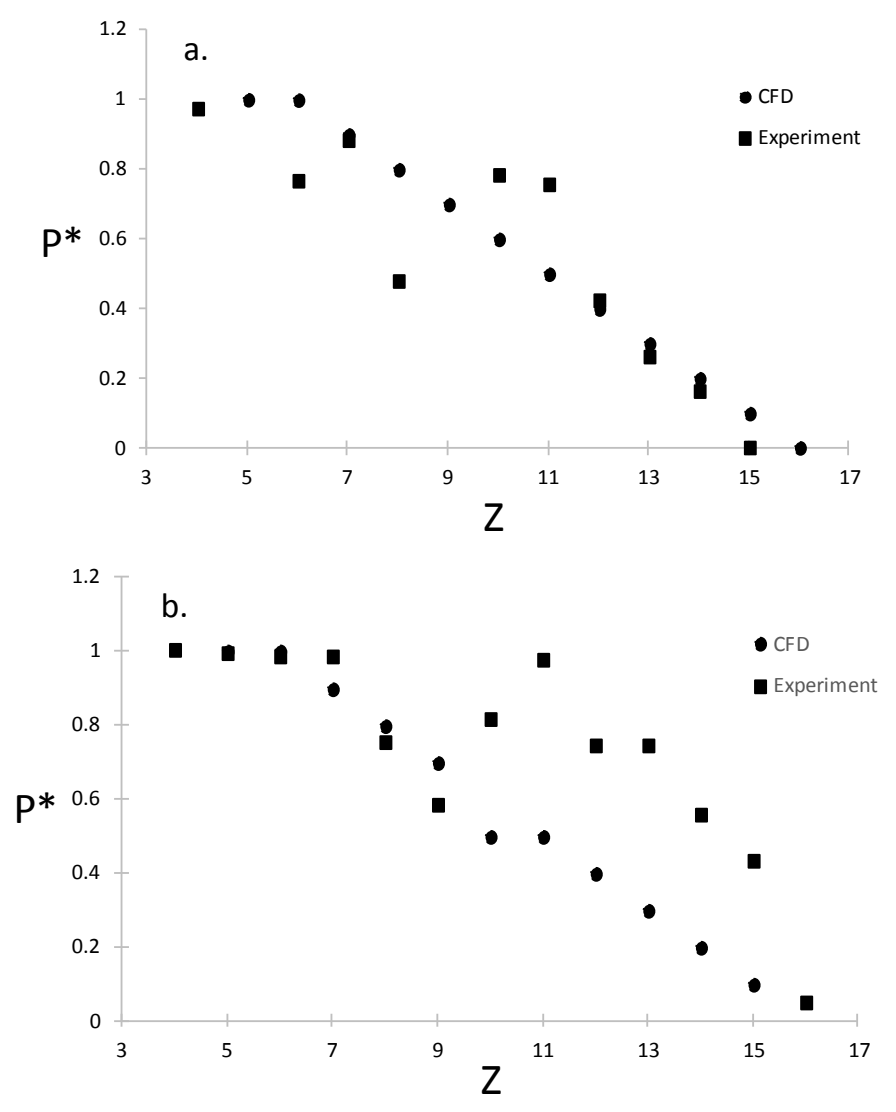

Figure 2.3.8. Comparisons of axial pressure distributions between the CFD model and experimental data from [12] for a. pressure ratios of 1.25-1.42 and b. ratios above 3.8.

most operating pressures five initial simulations were performed. If none of the permeability values resulted in a mass flow rate within $3 \%$ of the experimentally measured value, then another round of simulations were performed with the permeability guesses centered around the closest value from the previous set.

In order to calibrate the permeability at each operating pressure, the first step was to begin with an estimate based on previous values found in the literature (Dogu, 2005). From the value of the axial viscous resistance coefficient in Table 1, the initial guess value of permeability was found:

$$
\kappa=\frac{\mu}{B}=\frac{1.82 \times 10^{-5} \frac{\mathrm{kg}}{\mathrm{m} \cdot \mathrm{s}}}{4.5 \times 10^{7} \frac{\mathrm{kg}}{\mathrm{m}^{3} \cdot \mathrm{s}}}=4.044 \times 10^{-13} \mathrm{~m}^{2}
$$


This value was used to establish a range of permeability values for the first pressure ratio ( $R p$ $=1.194)$. The ranges for successive operating pressures were typically centered around the accepted best value from the previous point. On average, the permeability for each operating point was found from running 8 simulations, each requiring approximately 3.5 hours to converge. The computation time for this study was therefore approximately 392 hours. This was made feasible by the use of parallel computing on the Rivanna Cluster at the University of Virginia.

\subsubsection{Results of Permeability Variation Study}

The results of the CFD simulations are presented in Table 2.3.2 and Figure 2.3.8. For pressure ratios up to 2.5 it was found that the permeability of the bristle pack was estimated to decrease with increasing pressure ratio. At pressure ratios above 2.5 , however, it was found that estimated permeability values were relatively constant. This was perhaps due to the bristle pack reaching a compaction threshold as pressure ratios approached a critical value of 2.5 . It is worth noting that over this range of pressure the permeability changed by nearly an order of magnitude. The percentage difference between the maximum and minimum permeability values is $\sim 75 \%$.

\begin{tabular}{|c|c|}
\hline \multicolumn{2}{|c|}{ Pressure Ratio Permeability $\left.\mathbf{~ m}^{\mathbf{2}}\right]$} \\
\hline 1.194 & $1.36 \mathrm{E}-12$ \\
\hline 1.323 & $9.40 \mathrm{E}-13$ \\
\hline 1.361 & $1.2 \mathrm{E}-12$ \\
\hline 1.89 & $8 \mathrm{E}-13$ \\
\hline 2.123 & $7.3 \mathrm{E}-13$ \\
\hline 2.342 & $6.5 \mathrm{E}-13$ \\
\hline 2.471 & $6.8 \mathrm{E}-13$ \\
\hline 2.639 & $6.3 \mathrm{E}-13$ \\
\hline 2.69 & $7.4 \mathrm{E}-13$ \\
\hline 2.923 & $6.4 \mathrm{E}-13$ \\
\hline 3.142 & $7 \mathrm{E}-13$ \\
\hline 3.4 & $7.5 \mathrm{E}-13$ \\
\hline 3.697 & $7.2 \mathrm{E}-13$ \\
\hline 3.994 & $6.2 \mathrm{E}-13$ \\
\hline
\end{tabular}

Table 2.3.2 Values of bristle pack permeability. 


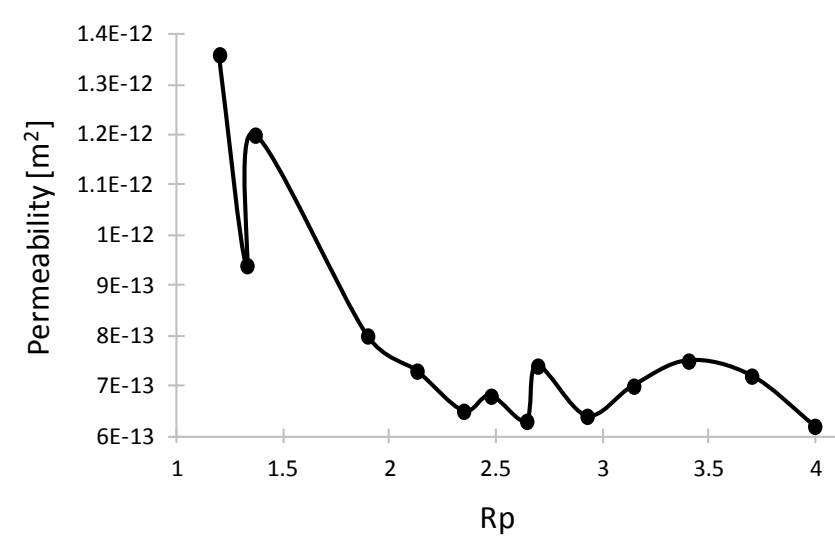

Figure 2.3.8. Experimental and calculated values of brush seal permeability vs. pressure ratio.

While the overall trend is a decrease in permeability with increasing pressure ratio, there are some local fluctuations and regions where the permeability increases with increasing pressure ratio. It is possible that this is an erroneous effect due to measurement error in the experimental data. If that is the case then the general flattening of the data above a pressure ratio of 2.5 would indicate this as the critical pressure ratio. Another potential explanation would be that temporary deformation or interaction between the bristles occurred when the data was originally measured. Because the simulations in this study did not have any physical model for the behavior of the bristles - but only for their effective porous behavior - this cannot be verified.

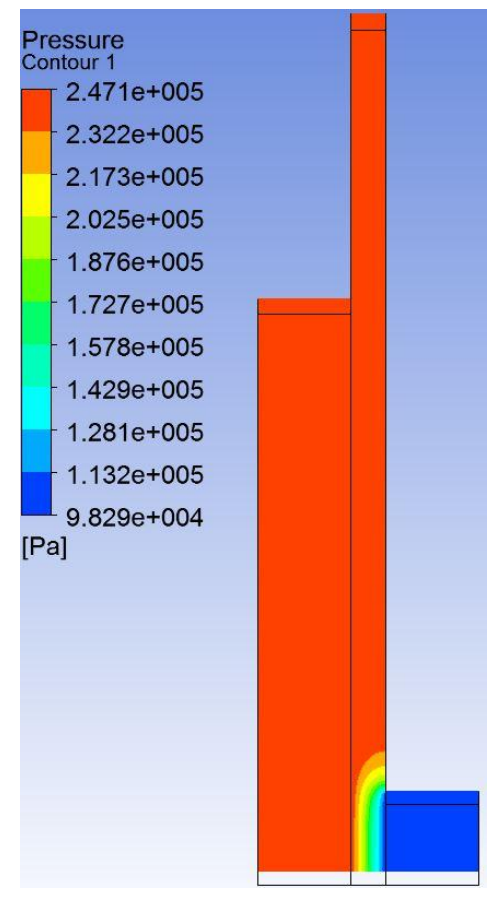

Figure 2.3.9. Pressure contour plot at seal mid-plane. 


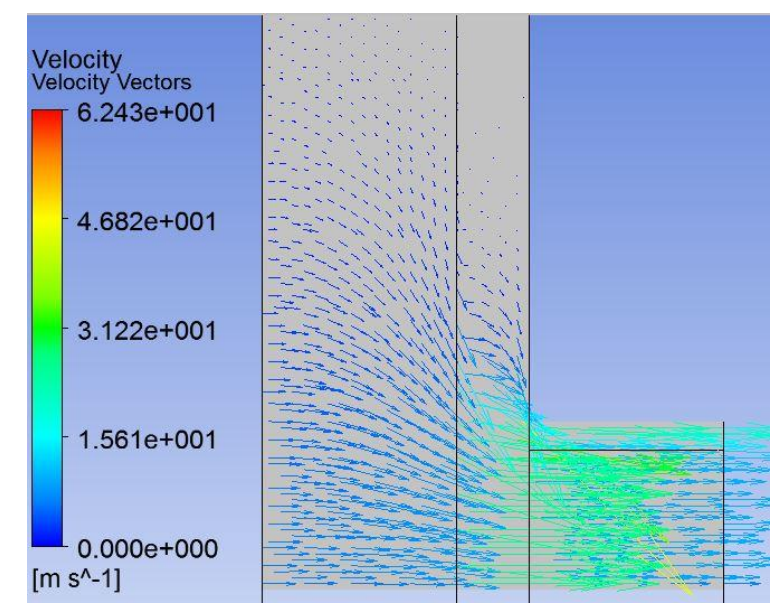

Figure 2.3.10. Velocity vector plot at seal mid-plane.

\subsubsection{Comparison With Darcy's Law}

Simply using Darcy's law to calculate the permeability at every point does give values for the permeability at each pressure ratio. However, doing this gives different values of permeability than the ones found from the iterative CFD method presented in this section. The charts below demonstrate that direct calculations from Darcy's law give a range of permeability that is not of the same order of magnitude (see Fig. 2.3.11a), likely due to a lack of consideration of compaction effects. For the sake of comparing the relative changes in permeability from this study to those predicted by a direct application of Darcy's Law, the two sets of permeability can both be nondimensionalized by dividing each value by the smallest value in the set. It now becomes clear that no major discrepancy exists in the prediction of changes in permeability, despite the differences in predicted value of the permeability magnitude (see Fig. 2.3.11b).

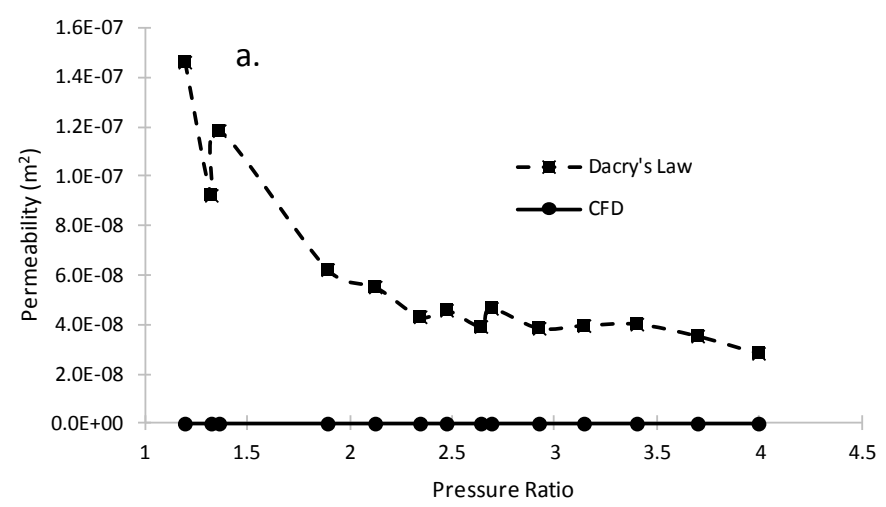




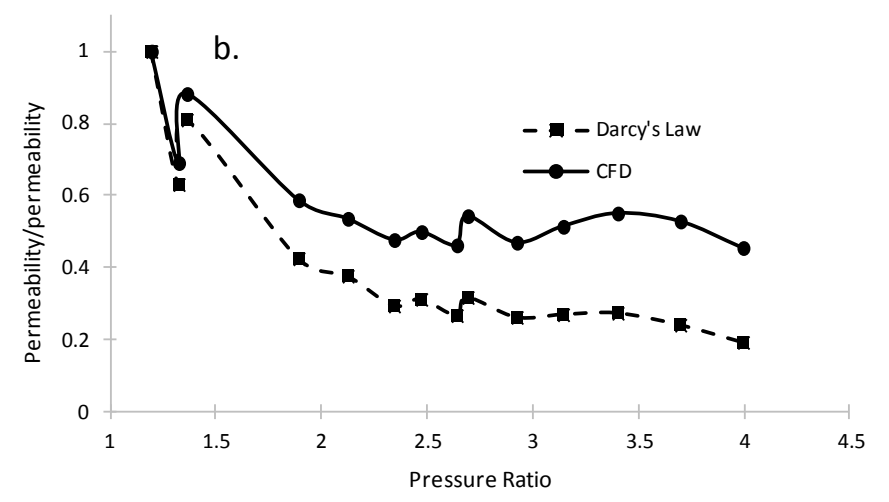

Figure 2.3.11. Comparison of permeability calculated directly from Darcy's law with permeability found using CFD a. permeability in units of $\mathrm{m}^{2}$ b. nondimensionalized permeability.

Modern research in porous media diffusion has indicated that characterizing permeability in terms of tortuosity will provide more insightful models for predicting flow behavior. In general terms, the tortuosity is the ratio of the effective hydraulic path length to the straight line distance in the direction of flow (Guo, 2012). Although there are many different proposed ways to describe a medium's tortuosity, it would be difficult even in theory to measure tortuosity. However, the concept is useful for providing qualitative understanding because it is inversely proportional to permeability (Nolen-Hoeksema, 2014).

It is somewhat intuitive that higher internal tortuosity would result in a less permeable material. The shortened flow path between individual bristles would result in fluid particles passing more slowly through the pack. In order to characterize this in a formal mathematical sense, it would be necessary to account for the dynamics of bristle deformation and interaction under pressure force loadings.

\subsubsection{Inertial Effects}

In the results displayed above, the flow behavior was assumed to be dominated by viscous effects. However, in order to test this assumption, three simulations were chosen to be run without neglecting the inertial effects. The three cases tested were those with pressure ratios of 1.19355, 2.47097, and 3.99355. These values were chosen because they represent the lowest, middle, and highest pressure ratios. The inertial permeability in the axial direction was initially set by using the value calculated in [9]. When the inertial term was included in the CFD model the maximum change in the mass flow through the seal was less than $0.001 \%$. The inertial permeability was then varied between $10 \mathrm{e}-12 \mathrm{~m}^{2}$ and $10 \mathrm{e} 12 \mathrm{~m}^{2}$. Despite this wide variation in the values of inertial permeability, the change in mass flow rate through the seal was never altered by more than $2 \%$ from the value found by using the corresponding non-inertial model.

\subsubsection{Conclusions From Permeability Variation Study}

In this section, experimental data of brush seal performance was used along with an extensive set of CFD simulations to study the changes in the permeability of a brush seal bristle pack as pressure loading was increased. It was demonstrated that the effective permeability of the bristle pack undergoes a significant reduction when the ratio of inlet to outlet pressure is increased, 
eventually reaching a relatively constant value perhaps due to the bristle pack reaching a threshold for compaction. While the effects of bristle deformation and shape were not explored in this study, they can be of significance to permeability. With this knowledge of the tendency for permeability to change with operating pressure, investigation of anisotropic permeability models and non-Darcian flow models are considered later in this thesis.

\section{Chapter 3 \\ Model Development}

\subsection{Governing Equations}

\subsubsection{Incompressible Fluids}

As discussed in section 2.1, the general equation to model flow through a porous medium is given by the Darcy-Forchheimer law (equation 2.1.4), and under conditions where inertial effects are minimal Darcy's law is considered sufficient (equation 2.1.3). The general three dimensional form of Darcy's can be expressed by writing equation 2.1.3 in component form:

$$
\frac{\partial p}{\partial x}=-\frac{\mu}{\kappa_{x}} u, \quad \frac{\partial p}{\partial y}=-\frac{\mu}{\kappa_{y}} v, \quad \frac{\partial p}{\partial z}=-\frac{\mu}{\kappa_{z}} w
$$

For two-dimensional flow, we are only concerned with axial (x-direction) and radial (ydirection) flow. The set of equations now reduces to:

$$
\frac{\partial p}{\partial x}=-\frac{\mu}{\kappa_{x}} u, \quad \frac{\partial p}{\partial y}=-\frac{\mu}{\kappa_{y}} v
$$

Conservation of mass within the bristle pack can be expressed in terms of the continuity equation:

$$
\frac{\partial \rho}{\partial t}+\frac{\partial(\rho u)}{\partial x}+\frac{\partial(\rho v)}{\partial y}+\frac{\partial(\rho w)}{\partial z}=0
$$

Again we restrict our focus to two dimensions, which allows equation 16 to be reduced to the following form: 


$$
\frac{\partial \rho}{\partial t}+\frac{\partial(\rho u)}{\partial x}+\frac{\partial(\rho v)}{\partial y}=0
$$

For typical turbomachinery applications, the flow through the stages of a compressor or turbine will experience a great deal of compression or expansion. However, for certain fluids (typically liquids) the flow through an individual seal may experience comparatively little change in thermodynamic properties. For such cases, the flow may be accurately modeled as incompressible. This assumption reduces the continuity equation to the following form:

$$
\frac{\partial u}{\partial x}+\frac{\partial v}{\partial y}=0
$$

Solving equation 15 for $u$ and $v$, and then substituting those expressions into equation 17 gives:

$$
\kappa_{x} \frac{\partial^{2} p}{\partial x^{2}}+\kappa_{x} \frac{\partial^{2} p}{\partial y^{2}}=0
$$

The mass flow rate through the seal can be calculated from the following surface integral:

$$
\dot{m}=\iint \rho \overrightarrow{\boldsymbol{V}} \cdot d \overrightarrow{\boldsymbol{A}}
$$

Which can be greatly simplified due to the cylindrical symmetry of this application and the assumption that effects of compressibility are negligible.

$$
\dot{m}=2 \pi \rho \int_{R_{i}}^{R_{o}} u(r) \cdot r d r
$$

The evaluation of this integral is equivalent to the summation of the flow rate through an infinite number of annuli of infinitesimal thickness and with radii varying from the inner radius of the seal to the outer radius. This technique is sometimes referred to as the "washer method" in standard calculus textbooks.

\subsubsection{Compressible Fluids}

Brush seals are commonly used in applications where the fluid to be sealed is a gas moving at relatively high speeds or undergoing large changes in pressure. For example, situations where air is moving with a Mach number of approximately 0.3 , the change in density is expected to be greater than $5 \%$ and compressibility effects are no longer negligible.

For applications involving compressible flow, the governing equations of section 3.1.1 are not valid. By considering density as a variable rather than a constant, it will no longer cancel out of the continuity equation. 


$$
\frac{\partial}{\partial x}\left(\rho(x, y)\left(\frac{\kappa_{x}}{\mu} \frac{\partial p}{\partial x}\right)\right)+\frac{\partial}{\partial y}\left(\rho(x, y)\left(\frac{\kappa_{y}}{\mu} \frac{\partial p}{\partial y}\right)\right)=0
$$

Where density is written as a function of $\mathrm{x}$ and $\mathrm{y}$. expanding the derivative and simplifying gives:

$$
\kappa_{x}\left[\frac{\partial \rho}{\partial x} \frac{\partial p}{\partial x}+\rho \frac{\partial^{2} p}{\partial x^{2}}\right]+\kappa_{y}\left[\frac{\partial \rho}{\partial y} \frac{\partial p}{\partial y}+\rho \frac{\partial^{2} p}{\partial y^{2}}\right]=0
$$

Equation 3.1.10 describes the pressure and density distribution throughout the plane of the bristle pack. Because pressure and density are interdependent, this is a non-linear PDE. The relationship between density and pressure must be defined through an equation of state, such as the ideal gas law.

While equations 3.1.6 and 3.1.10 are two dimensional, it would be possible to adapt this method to three-dimensional brush seal modeling. This would allow for the incorporation of flow field effects induced by shaft rotation. A three-dimensional (e.g. cylindrical) coordinate system could be established in order to appropriately express Darcy's law and continuity.

\subsection{Domain and Boundary Conditions}

The domain is defined as a plane which extends in the axial direction from the front plate to the back plate (see Fig. 3.2). The bristle pack is considered to be a porous medium. The rotor, stator, front plate, and back plate are wall boundaries, and the inlet and outlet are constant pressure boundaries. It is assumed that the bristles are in contact with the rotor, and are of the same material properties throughout the region (homogeneous porous medium).

The inlet and outlet, which will also be referred to as the upstream and downstream pressure boundaries, respectively, are subjected to prescribed pressure values $\mathrm{Pu}_{\mathrm{u}}$ and $\mathrm{P}_{\mathrm{d}}$. These are Dirichlet boundary conditions. At the rotor, stator, front plate and back plate there cannot be any flow across the boundary. This corresponds to Neumann boundary conditions:

$$
\begin{gathered}
\frac{\partial p}{\partial x}=0 \quad\left(x=0, y>y_{f p}\right) \text { and }\left(x=X, y>y_{b p}\right) \\
\frac{\partial p}{\partial y}=0 \quad(x, y=0) \text { and }(x, y=Y) \\
p=P_{u} \quad\left(x=0, y<y_{f p}\right) \text { and }\left(x=X, y<y_{b p}\right)
\end{gathered}
$$




$$
p=P_{d} \quad\left(x=0, y<y_{f p}\right) \text { and }\left(x=X, y<y_{b p}\right)
$$

Where $y_{f p}$ is the $y$ coordinate of the bottom of the front plate, $y_{b p}$ is the $y$ coordinate of the bottom of the back plate, $X$ is the overall axial length of the seal, and $Y$ is the overall radial length of the seal.

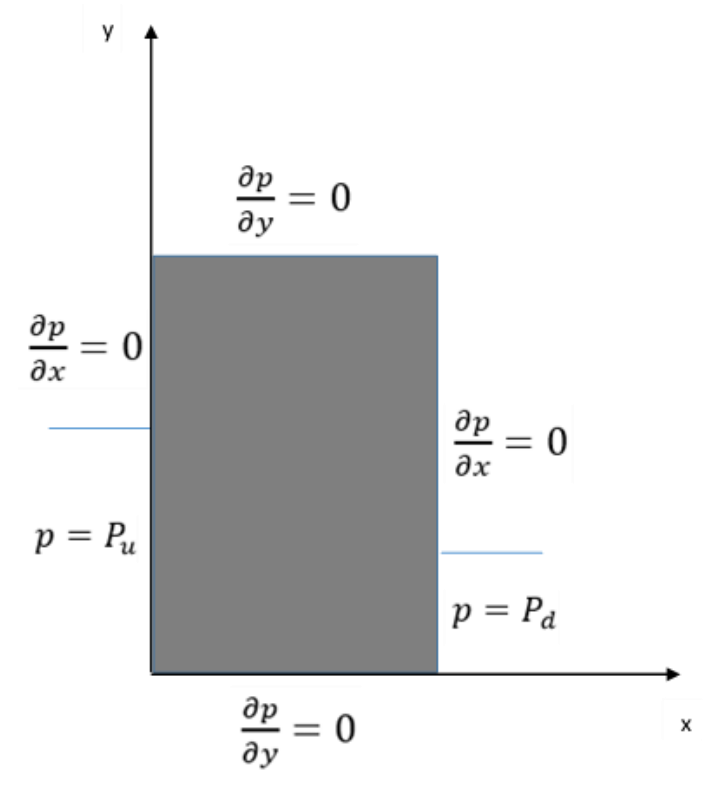

Figure 3.2. Model Domain.

For a typical brush seal, the radial length is approximately 15 to 25 times greater than the axial length (Chew, Lapworth, \& Millener, Mathematical modeling of brush seals, 1995).

\subsection{Analytical Methods}

While the primary focus of this thesis is the development of a numerical method for calculating the pressure distribution in a brush seal, a brief discussion of analytical solution techniques for the compressible flow within the bristle pack is given here. Equation 3.1.6 is a linear, second order elliptic partial differential equation. The coordinate system can be redefined by normalizing $x$ and $y$ by $\sqrt{\kappa_{x}}$ and $\sqrt{\kappa_{y}}$, respectively. The chain rule can be applied to obtain expressions for the partial derivatives of pressure:

$$
\tilde{x}=\frac{x}{\sqrt{\kappa_{x}}}
$$




$$
\begin{gathered}
\frac{\partial p}{\partial x}=\frac{\partial p}{\partial \tilde{x}} \frac{\partial \tilde{x}}{\partial x}=\frac{1}{\sqrt{k_{x}}} \frac{\partial p}{\partial \tilde{x}} \\
\frac{\partial^{2} p}{\partial x^{2}}=\frac{\partial}{\partial \tilde{x}}\left(\frac{\partial p}{\partial x}\right) \frac{1}{\sqrt{\kappa_{x}}}=\frac{1}{\kappa_{x}} \frac{\partial p}{\partial \tilde{x}}
\end{gathered}
$$

The same procedure can be repeated for $y$, resulting in:

$$
\frac{\partial^{2} p}{\partial y^{2}}=\frac{1}{\kappa_{y}} \frac{\partial p}{\partial \widetilde{y}}
$$

These expressions for the second partial derivatives of pressure can be substituted into equation 3.1.6. The result is Laplace's equation in terms of the variables $\tilde{x}$ and $\tilde{y}$ :

$$
\frac{\partial^{2} p}{\partial \tilde{x}^{2}}+\frac{\partial^{2} p}{\partial \tilde{y}^{2}}=\nabla^{2} p=0
$$

The primary method to solve problems of this form is to assume the function $p(\tilde{x}, \tilde{y})$ is equal to the product of a function of strictly $\tilde{x}$ and a function of strictly $\tilde{y}$ :

$$
p(\tilde{x}, \tilde{y})=\tilde{X}(\tilde{x}) \tilde{Y}(\tilde{y})
$$

By substituting this expression for $p$ into Laplace's equation and separating the variables, a set of ordinary differential equations are formed:

$$
\begin{aligned}
& \tilde{X}^{\prime \prime}-\lambda^{2} \tilde{X}=0 \\
& \tilde{Y}^{\prime \prime}+\lambda^{2} \tilde{Y}=0
\end{aligned}
$$

Where $\lambda$ is called the "separation constant" and is an eigenvalue of the corresponding eigenfunctions $\tilde{X}$ and $\tilde{Y}$. These equations can then be solved for $\lambda=0$ and $\lambda \neq 0$. The solution to $p(\tilde{x}, \tilde{y})$ is the sum of the solutions for both cases. The final form of the exact solution depends on the boundary conditions. For certain cases (such as the Dirichlet problem) the solution may be expressed in terms of a Fourier series.

The present problem has mixed (Robin) boundary conditions, and discontinuous boundary conditions. These are described in section 3.2. The exact solution to Laplace's equation in this scenario is difficult to obtain, and extremely difficult to implement practically (Greenberg, 1998).

For the case of compressible flow, the governing equation is more complex. Depending on the specified relationship between pressure and density, the equation can become nonlinear. While there are methods for solving nonlinear partial differential equations, the solution techniques discussed in this thesis are limited to the numerical methods described in section 3.4.5. 


\subsection{Numerical Methods}

\subsubsection{Discretization of the Governing Equations - Incompressible}

For the incompressible case, applying a central difference scheme to approximate both of the $2^{\text {nd }}$ order derivatives in equation 3.1 .6 gives:

$$
\kappa_{x} D_{x, 0}^{2}(p)+\kappa_{y} D_{y, 0}^{2}(p)=0
$$

Where $D_{x, 0}^{2}$ represents the central difference operator applied with respect to $x$, and $D_{y, 0}^{2}$ represents the central difference operator applied with respect to y. Applying these approximations results in the difference equation:

$$
\begin{gathered}
\frac{\kappa_{x}}{\Delta x^{2}}\left(p_{i-1, j}-2 p_{i, j}+p_{i+1, j}\right)+\frac{\kappa_{y}}{\Delta y^{2}}\left(p_{i, j-1}-2 p_{i, j}+p_{i, j+1}\right)=0 \\
-2\left(\kappa_{x} \Delta y^{2}+\kappa_{y} \Delta x^{2}\right) p_{i, j}+\kappa_{x} \Delta y^{2}\left(p_{i-1, j}+p_{i+1, j}\right)+\kappa_{y} \Delta x^{2}\left(p_{i, j-1}+p_{i, j+1}\right)=0
\end{gathered}
$$

This can be written in a more compact form by defining parameters $\alpha, \beta$, and $\gamma$ :

$$
\alpha=\kappa_{x} \Delta y^{2}, \beta=\kappa_{y} \Delta x^{2}, \gamma=-2(\alpha+\beta)
$$

The scheme is therefore:

$$
\gamma p_{i, j}+\alpha\left(p_{i-1, j}+p_{i+1, j}\right)+\beta\left(p_{i, j-1}+p_{i, j+1}\right)=0
$$

In a similar manner, a central difference scheme may be applied to approximate the first derivatives in equation 3.1.2:

$$
\begin{gathered}
D_{x, 0}(p)=-\frac{\mu}{\kappa_{x}} u, \quad D_{y, 0}(p)=-\frac{\mu}{\kappa_{y}} v \\
-\left(\frac{p_{i+1, j}-p_{i-1, j}}{2 \Delta x}\right)=\frac{\mu}{\kappa_{x}} u_{i, j}, \quad-\left(\frac{p_{i, j-1}-p_{i, j+1}}{2 \Delta y}\right)=\frac{\mu}{\kappa_{y}} v_{i, j} \\
u_{i, j}=\frac{\kappa_{x}}{\mu}\left(\frac{p_{i-1, j}-p_{i+1, j}}{2 \Delta x}\right), \quad v_{i, j}=\frac{\kappa_{y}}{\mu}\left(\frac{p_{i, j-1}-p_{i, j+1}}{2 \Delta y}\right)
\end{gathered}
$$

The equations of 3.4.8 along with equation 3.4.5 form a system of three equations and three unknowns. Of course, the boundary conditions are given in terms of pressure, so the numerical 
solution will first calculate an approximation of the pressure field. Once the pressure field has been evaluated it is trivial to find the axial and radial velocity by applying 3.4.8.

The mass flow rate through the seal can be calculated numerically from the axial velocity by applying equation 3.1.8 at the outlet of the seal, and approximating the integral using the trapezoidal rule:

$$
\dot{m}=\pi \rho \Delta y \sum_{y=0}^{y=Y}\left[u\left(X, y_{i+1}\right) \cdot y_{i+1}+u\left(X, y_{i}\right) \cdot y_{i}\right]
$$

\subsubsection{Discretization of the Governing Equations - Compressible}

In the same manner, the equation for compressible flow can be discretized by applying finite difference approximations of the derivatives in equation 3.1.10:

$$
\begin{gathered}
\kappa_{x}\left[D_{x, 0}(\rho) D_{x, 0}(p)+\rho D_{x, 0}^{2}(p)\right]+\kappa_{y}\left[D_{y, 0}(\rho) D_{y, 0}(p)+\rho D_{y, 0}^{2}(p)\right]=0 \\
-2\left(\kappa_{x} \Delta y^{2}+\kappa_{y} \Delta x^{2}\right) p_{i, j}+\kappa_{x} \Delta y^{2}\left(\rho_{i, j}+\frac{\Delta x}{2}\left(D_{x, 0}(\rho)\right)\right) p_{i+1, j}+\kappa_{x} \Delta y^{2}\left(\rho_{i, j}-\frac{\Delta x}{2}\left(D_{x, 0}(\rho)\right)\right) p_{i-1, j} \\
+\kappa_{y} \Delta x^{2}\left(\rho_{i, j}+\frac{\Delta y}{2}\left(D_{y, 0}(\rho)\right)\right) p_{i, j+1}+\kappa_{y} \Delta x^{2}\left(\rho_{i, j}-\frac{\Delta y}{2}\left(D_{y, 0}(\rho)\right)\right) p_{i, j-1}=0
\end{gathered}
$$

This equation can be written in a more compact form by re-writing the coefficients of $p$ as:

$$
\begin{aligned}
& \gamma=-2\left(\kappa_{x} \Delta y^{2}+\kappa_{y} \Delta x^{2}\right) \\
& \alpha_{1}=\kappa_{x} \Delta y^{2}\left(\rho_{i, j}+\frac{\Delta x}{2}\left(D_{x, 0}(\rho)\right)\right) \\
& \alpha_{2}=\kappa_{x} \Delta y^{2}\left(\rho_{i, j}-\frac{\Delta x}{2}\left(D_{x, 0}(\rho)\right)\right) \\
& \beta_{1}=\kappa_{y} \Delta x^{2}\left(\rho_{i, j}+\frac{\Delta y}{2}\left(D_{y, 0}(\rho)\right)\right) \\
& \beta_{2}=\kappa_{y} \Delta x^{2}\left(\rho_{i, j}-\frac{\Delta y}{2}\left(D_{y, 0}(\rho)\right)\right)
\end{aligned}
$$

Equation 3.4.11 can now be written as:

$$
\gamma p_{i, j}+\alpha_{1} p_{i+1, j}+\alpha_{2} p_{i-1, j}+\beta_{1} p_{i, j+1}+\beta_{2} p_{i, j-1}=0
$$




\subsubsection{Implicit Solution Method}

In this section, an overview of the development of an implicit numerical solution method is given for the incompressible case. The treatment of compressible flow is saved for the section on explicit solutions. This is because the code which was developed (described in chapter 4) used only an explicit technique to handle compressible flow.

The discretized governing equations can be solved by employing an implicit method. Consider equation 3.4.5 applied along the columns of the pressure domain matrix:

$$
\begin{gathered}
\gamma p_{1,1}+\alpha\left(p_{0,1}+p_{2,1}\right)+\beta\left(p_{1,0}+p_{1,2}\right)=0 \\
\gamma p_{2,1}+\alpha\left(p_{1,1}+p_{3,1}\right)+\beta\left(p_{2,0}+p_{2,2}\right)=0 \\
\gamma p_{3,1}+\alpha\left(p_{2,1}+p_{4,1}\right)+\beta\left(p_{3,0}+p_{3,2}\right)=0 \\
\cdot \\
\cdot \\
\cdot \\
\gamma p_{N, 1}+\alpha\left(p_{N-1,1}+p_{N+1,1}\right)+\beta\left(p_{N, 0}+p_{N, 2}\right)=0 \\
\cdot \\
\cdot \\
\cdot \\
\gamma p_{N, N}+\alpha\left(p_{N-1, N}+p_{N+1, N}\right)+\beta\left(p_{N, N-1}+p_{N, N+1}\right)=0
\end{gathered}
$$

This set of equations can be expressed in matrix form as:

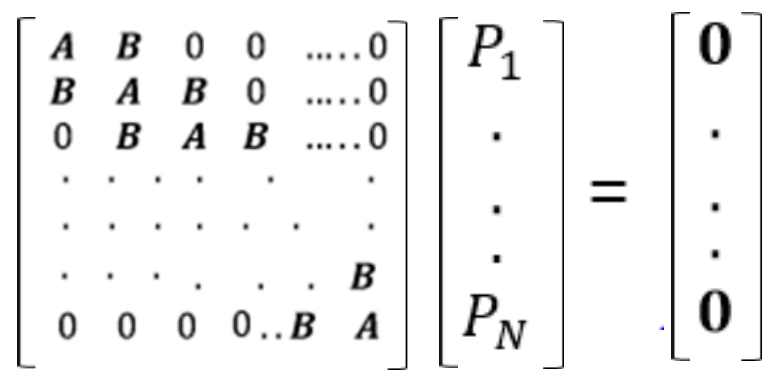

Where the matrix on the left is block tridiagonal, composed of the tridiagonal matrix $\mathbf{A}$ and the diagonal matrix B: 


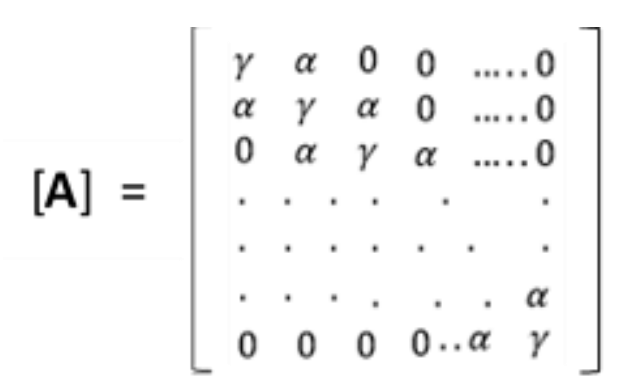

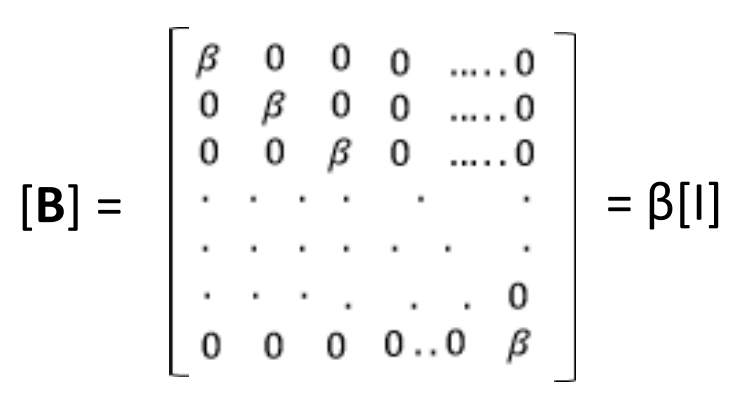

And the vector $\mathbf{P}$ is made up of sub-vectors which form the columns of the pressure domain matrix. For example, $\mathbf{P}_{\mathbf{1}}$ represents the pressure at each node along the first column:

$$
\left[\boldsymbol{P}_{1}\right]=\left[\begin{array}{c}
p_{1,1} \\
\cdot \\
\cdot \\
p_{N, 1}
\end{array}\right]
$$

The boundary conditions must be implemented in equation 3.4 .19 by modifying the matrices $\mathbf{A}$ and $\mathbf{B}$ for certain entries of the larger matrix. The system can then be solved computationally using a decomposition method.

The matrix that is formed in equation 3.4.19 becomes extremely large for refined meshes. If the overall mesh is chosen to contain NxN elements, then the matrix dimensions will be $\mathrm{N}^{2} \mathrm{xN}^{2}$.

\subsubsection{Explicit Solution Method - Incompressible Flow}

An explicit method can be implemented by iteratively solving for each column of the pressure domain matrix. First equation 3.4 .5 is written for the iteration step $n$ :

$$
\gamma p_{i, j}^{n}+\alpha\left(p_{i-1, j}^{n}+p_{i+1, j}^{n}\right)+\beta\left(p_{i, j-1}^{n}+p_{i, j+1}^{n}\right)=0
$$


This discreet equation gives the relationship between the pressure $p_{i, j}$ at one node and the pressure at the neighboring nodes. The value of the pressure at a given node for the iteration step $n+1$ can now be determined:

$$
p_{i, j}^{n+1}=-\frac{\alpha}{\gamma}\left(p_{i-1, j}^{n}+p_{i+1, j}^{n}\right)-\frac{\beta}{\gamma}\left(p_{i, j-1}^{n}+p_{i, j+1}^{n}\right)
$$

This is commonly known as the Jacobi iteration method. The convergence rate can be improved by altering the scheme to simultaneously solve for all nodes along a given dimension:

$$
\begin{aligned}
& p_{i, j}^{n+1}=-\frac{\alpha}{\gamma}\left(p_{i-1, j}^{n+1}+p_{i+1, j}^{n+1}\right)-\frac{\beta}{\gamma}\left(p_{i, j-1}^{n}+p_{i, j+1}^{n}\right) \\
& \frac{\alpha}{\gamma} p_{i-1, j}^{n+1}+p_{i, j}^{n+1}+\frac{\alpha}{\gamma} p_{i+1, j}^{n+1}=-\frac{\beta}{\gamma}\left(p_{i, j-1}^{n}+p_{i, j+1}^{n}\right)
\end{aligned}
$$

In this case, the dimension correspond to $\mathrm{j}$ was chosen. This is known as the line method, and all values of the solution along a given row (or column) are computed simultaneously. It can be reformulated as:

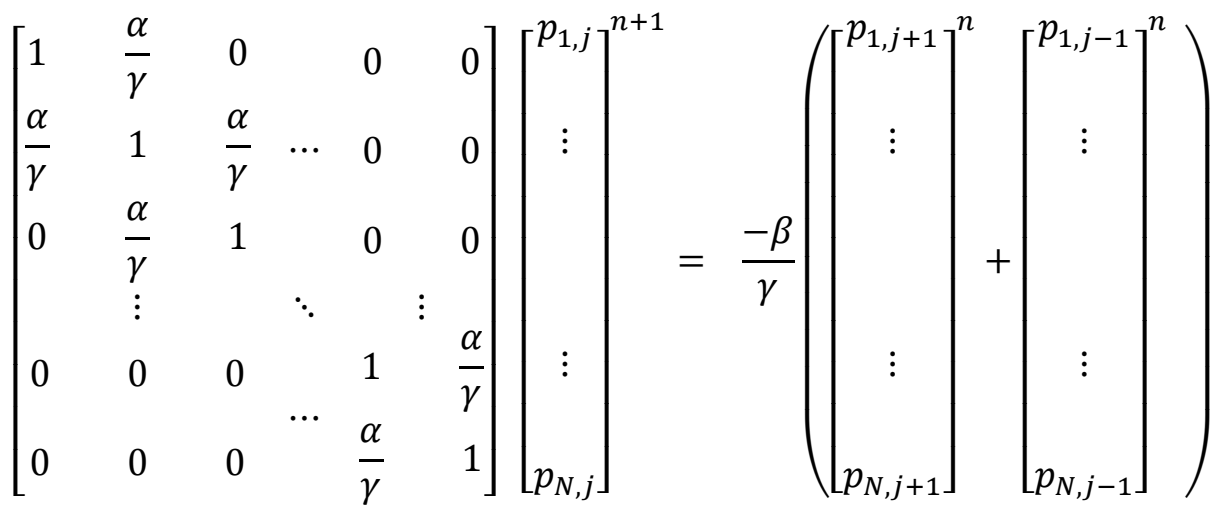

Where the pressure values on the right side of the equation are from the previous iteration. When this method is used in an algorithm a "sweep" is performed in the i direction to calculate each line of constant $\mathrm{j}$. 


\subsubsection{Explicit Solution Method - Compressible Flow}

In a similar manner, equation 3.4.18 can be solved along a line. If values of constant $j$ are moved to one side of the equation, and the values on the other side are taken from the previous iteration, the scheme becomes:

$$
\frac{\alpha_{2}}{\gamma} p_{i-1, j}^{n+1}+p_{i, j}^{n+1}+\frac{\alpha_{1}}{\gamma} p_{i+1, j}^{n+1}=\frac{-\beta_{1}}{\gamma} p_{i, j+1}^{n}+\frac{-\beta_{2}}{\gamma} p_{i, j-1}^{n}
$$

This scheme can also be written in matrix form:

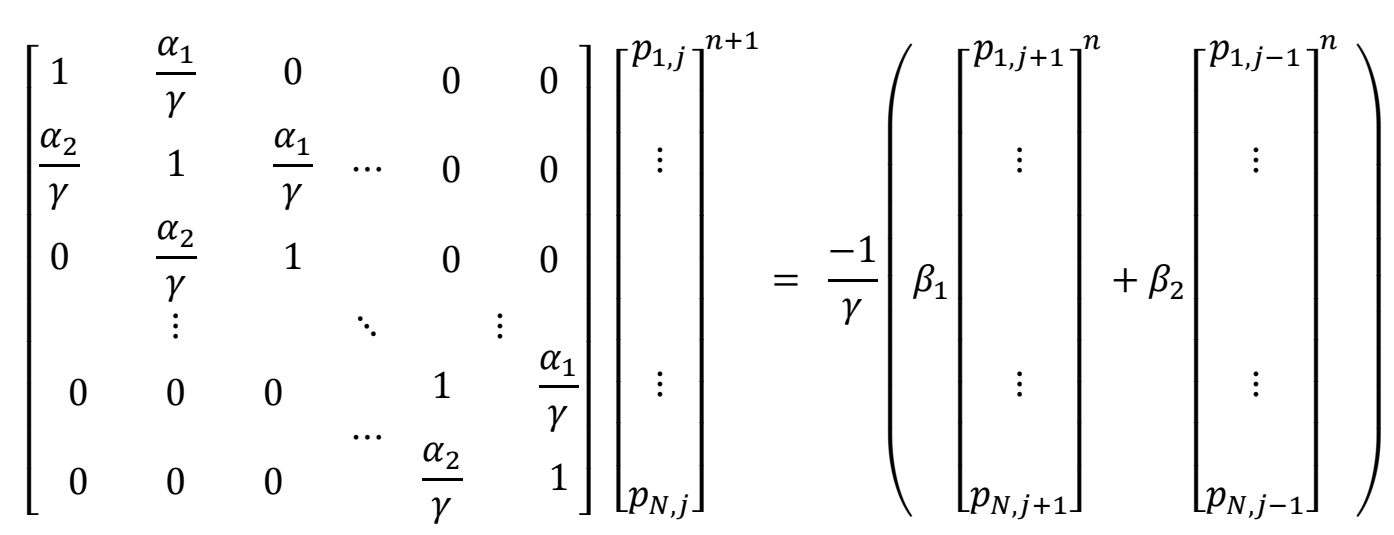

Where the quantities $\alpha_{1}, \alpha_{2}, \beta_{1}, \beta_{2}$ are vectors which are dependent on density and its partial derivatives, as described in section 3.4.2. An important feature of this explicit method is that it treats density as a variable.

A relaxation factor $\omega$ can be defined for the schemes shown in equations 3.4.27 and 3.4.30. After each sweep has been performed to calculate the Jacobi iterate, the updated solution is found from the Jacobi iterate and the solution from the previous iteration:

$$
p_{i, j}^{n+1}=\omega p_{i, j}^{*}+(1-\omega) p_{i, j}^{n}
$$

Where $p_{i, j}^{*}$ is the solution from the scheme at each iteration. Due to the nature of this scheme the relaxation factor should be chosen from the range $0<\omega \leq 1$. Choosing a relaxation factor less than 1 will slow the convergence rate, but can establish convergence for cases in which divergence would otherwise occur.

In order to have a closed system, an equation of state must relate density and pressure. For air under isothermal conditions, the ideal gas model can be used to provide the necessary relationship. The density at each node is easily found from equation 3.4.32: 


$$
\rho_{i, j}=\frac{p_{i, j}}{R T}
$$

The partial derivatives of density which are required for the scheme can be approximated by using the central difference method.

\subsection{Code Development}

While software packages capable of modeling general two and three dimensional porous media flow already exist, there is a need for a simple model, developed specifically for solving the pressure distribution within the bristle pack of a brush seal.

The methods described in this chapter were implemented to develop a finite-difference based computer program. This code is capable of accepting basic geometry and material properties, generating a sufficiently fine mesh, solving the system of numerical equations, and providing desired information of the flow within the seal. Results are based on two-dimensional calculations and are therefore limited to the analysis of an individual plane with dimensions in the axial and radial directions.

By solving for the two-dimensional pressure distribution, it is possible to also predict density distribution, velocity distribution, mass flow rate and aerodynamic forces acting on the bristles.

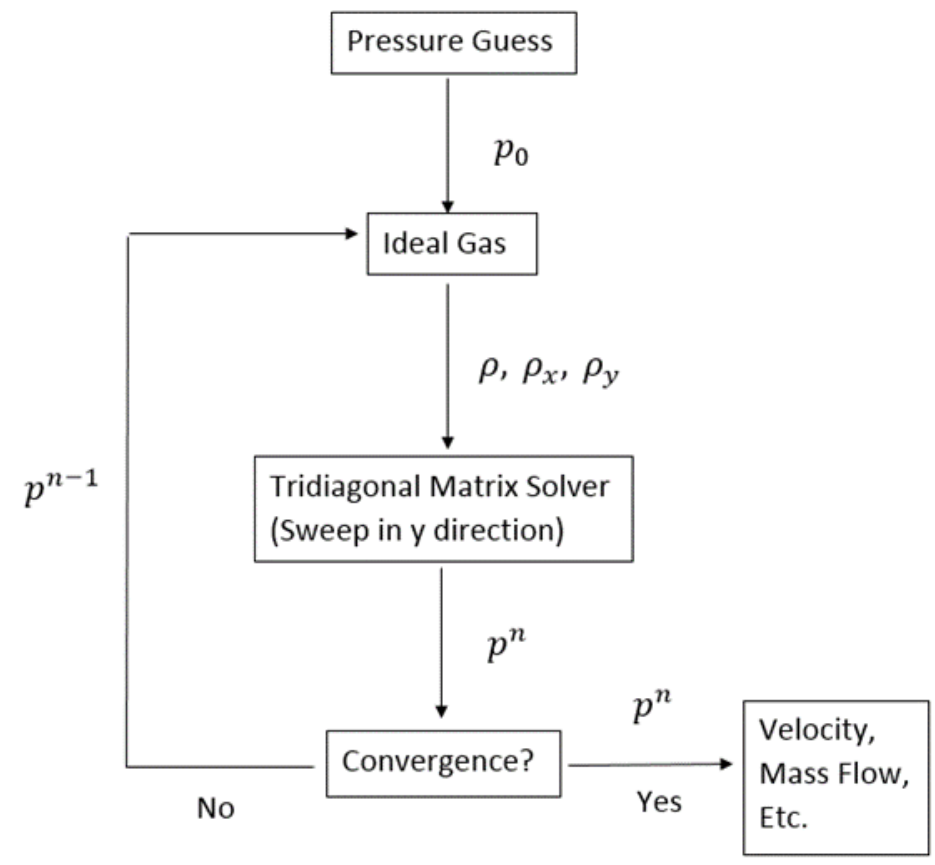

Fig. 3.5 Program algorithm 
Because the solution relies on information about the density field from the previous iteration, it is useful to "decouple" the density and pressure calculations. The algorithm shown in figure 3.5 shows the basic structure of the developed code.

\subsection{Code Assessment}

The two sources of error in a finite-difference scheme are machine error and discretization error. The machine error is associated with rounding during floating point calculations and must be considered, especially when values being stored require many significant figures (e.g. 16). The algorithm described in this thesis iteratively calculates and stores values of pressure in the standard SI unit system. Values are expected to be between the specified inlet and outlet boundary pressures calculated in Pa. Physically realistic values should be on the order of $10^{5} \mathrm{~Pa}-10^{7} \mathrm{~Pa}$.

It should be noted that permeability coefficients for the bristle pack, expressed in standard SI units, are expected to be on the order of $10^{-13} \mathrm{~m}^{2}$. However, when the scheme is implemented as described by equation 3.4.30 all terms containing permeability coefficients are divided by terms which are also multiples of permeability. Therefore, no values are expected to require a large amount of precision to maintain the required number of significant digits.

Code performance can be investigated by considering consistency, convergence, and stability. A numerical scheme for solving a PDE is considered consistent with the PDE if it approaches the exact solution as the discretization size approaches zero. For the problem of compressible flow, the PDE to be approximated is equation 3.1.10:

$$
\kappa_{x}\left[\frac{\partial \rho}{\partial x} \frac{\partial p}{\partial x}+\rho \frac{\partial^{2} p}{\partial x^{2}}\right]+\kappa_{y}\left[\frac{\partial \rho}{\partial y} \frac{\partial p}{\partial y}+\rho \frac{\partial^{2} p}{\partial y^{2}}\right]=0
$$

Where the scheme is defined by equation 3.4.11:

$$
\begin{gathered}
-2 \rho_{i, j}\left(\kappa_{x} \Delta y^{2}+\kappa_{y} \Delta x^{2}\right) p_{i, j}+\kappa_{x} \Delta y^{2}\left(\rho_{i, j}+\frac{\Delta x}{2}\left(D_{x, 0}(\rho)\right)\right) p_{i+1, j}+\kappa_{x} \Delta y^{2}\left(\rho_{i, j}-\frac{\Delta x}{2}\left(D_{x, 0}(\rho)\right)\right) p_{i-1, j} \\
+\kappa_{y} \Delta x^{2}\left(\rho_{i, j}+\frac{\Delta y}{2}\left(D_{y, 0}(\rho)\right)\right) p_{i, j+1}+\kappa_{y} \Delta x^{2}\left(\rho_{i, j}-\frac{\Delta y}{2}\left(D_{y, 0}(\rho)\right)\right) p_{i, j-1}=0
\end{gathered}
$$

The numerical derivatives $D_{x, 0}$ and $D_{y, 0}$ are central difference approximations, which are derived from the Taylor series expansion and are known to be $2^{\text {nd }}$ order accurate. Therefore as discretization size $\Delta x$ and $\Delta y$ approach zero linearly, the truncation error for each derivative approximation will approach zero quadratically. 
A scheme is considered stable if the error caused by a small perturbation in the numerical solution remains bounded. As demonstrated in chapter 3 , the entire scheme can be expressed in matrix form:

$$
[A]\{p\}=\{b\}
$$

Where the vector $\{p\}$ contains all nodes in the grid, the matrix $A$ is block tri-diagonal with the coefficient terms $\gamma, \alpha_{1}, \alpha_{2}, \beta_{1}, \beta_{2}$ from section 3.4.5, and the vector $\mathrm{b}$ incorporates the boundary conditions. The matrix $A$ can be expressed as a sum of a diagonal matrix and a "remainder matrix":

$$
[A]=[D]-[R]
$$

The iterative scheme can then be written as:

$$
[D]\{p\}^{n+1}=[R]\{p\}^{n}+\{b\}
$$

Let the exact solution be $p_{e}$, and let the error in the numerical scheme be $\varepsilon=p-p_{e}$, the exact solution can be substituted into the scheme:

$$
[D]\left\{p_{e}\right\}^{n+1}=[R]\left\{p_{e}\right\}^{n}+\{b\}
$$

By subtracting equation 3.6.4 from equation 3.6.3 and multiplying by the inverse of $D$, an expression for the error is obtained:

$$
\{\varepsilon\}^{n+1}=[D]^{-1}[R]\{\varepsilon\}^{n}
$$

The error will be bounded, and the equation will therefore be stable if the eigenvalues of the matrix $\left(D^{-1} R\right)$ are all less than 1 (Young, Iterative Solution of Large Linear Systems, 1971).

The code that was developed for this thesis was tested for a small mesh to demonstrate the stability of the scheme. The matrix $\left(D^{-1} R\right)$ changes for each iteration because it is a function of density, so the eigenvalues for each iteration were stored. The absolute value of the maximum eigenvalue is referred to as the spectral radius. 


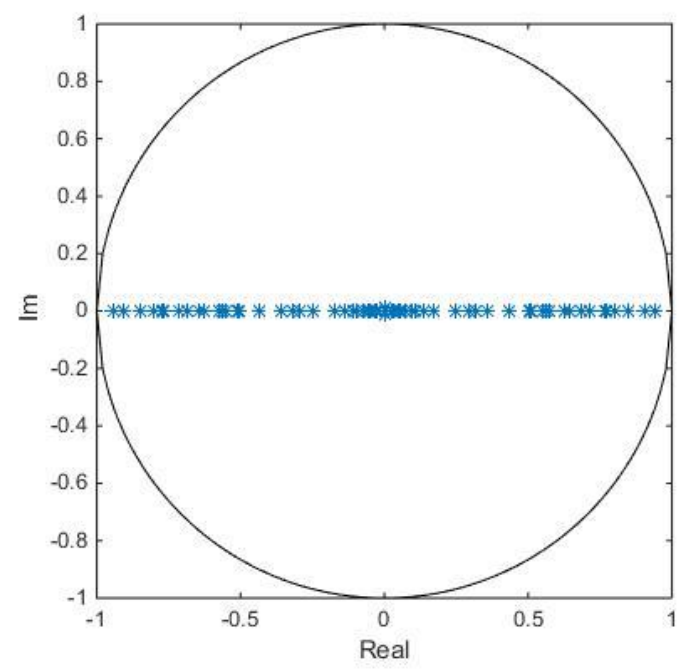

Figure 3.6.1 Plot of Eigenvalues

Fig. 3.6.1 demonstrates that the magnitude of all eigenvalues of the matrix $\left(D^{-1} R\right)$ are less than one. This is considered a standard criterion for scheme convergence.

\section{Chapter 4 \\ Results and Discussion}

\subsection{Mesh Independence Study}

An important aspect of CFD code performance is its ability to provide solutions that are not dependent on the grid density. For successive grid refinement, results are expected to asymptotically converge upon final values. To demonstrate mesh independence, several simulations were run for the same operating conditions and bristle properties. Results are considered to have convereged when the RMS residual of the pressure matrix is less than 1e-4. The details of the four meshes are provided in table 4.1. 


\begin{tabular}{|c|c|c|}
\hline Mesh Nodes & Iterations & Wall-Clock Time (s) \\
\hline 100 & 498 & 0.099159 \\
\hline 400 & 2163 & 0.63985 \\
\hline 2500 & 13800 & 12.375 \\
\hline 4900 & 26721 & 36.669 \\
\hline 8100 & 43349 & 89.632 \\
\hline 10000 & 52309 & 123.78 \\
\hline 14400 & 74020 & 222.35 \\
\hline
\end{tabular}

Table 4.1.1 Meshes for Mesh Independence Study

The set of operating parameters for testing mesh independence is given in table 4.2. These values were chosen because they are standard and within the range of typical seal testing. The fluid was air modeled as an ideal gas.

\begin{tabular}{|c|c|}
\hline Parameter & Value \\
\hline Axial Permeability & $2.40 \mathrm{E}-11 \mathrm{~m}^{2}$ \\
\hline Radial Permeability & $2.40 \mathrm{E}-08 \mathrm{~m}^{2}$ \\
\hline Temperature & $293.15(\mathrm{~K})$ \\
\hline Upstream Pressure & $169.677(\mathrm{Kpa})$ \\
\hline Downstream Pressure & $100(\mathrm{Kpa})$ \\
\hline
\end{tabular}

Table 4.1.2 Mesh Study Parameters

The results from this mesh study demonstrate that predicted mass flow and average fluid density approach final values as the mesh is increasingly refined. Figures 4.1 (a) and 4.1(b) show the mass flow rate and average fluid density versus the number of elements in the mesh. In order to maintain generality these values were non-dimensionalized by dividing each set by its minimum value. 


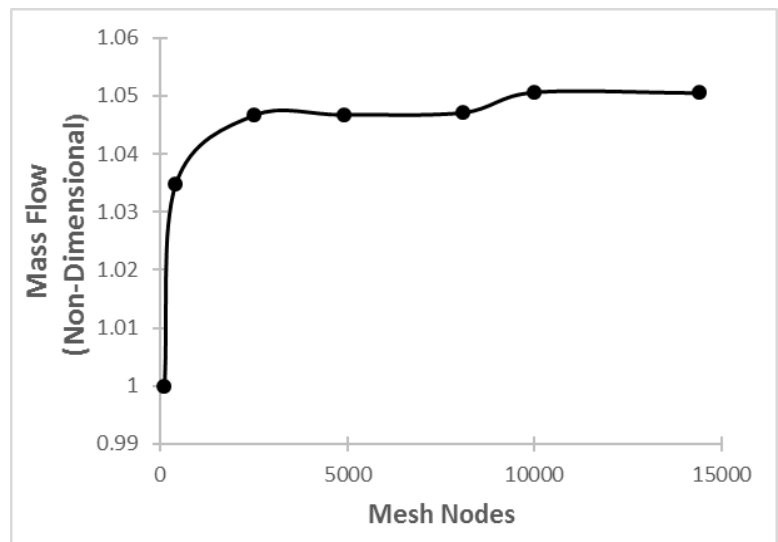

(a) Mass Flow Rate

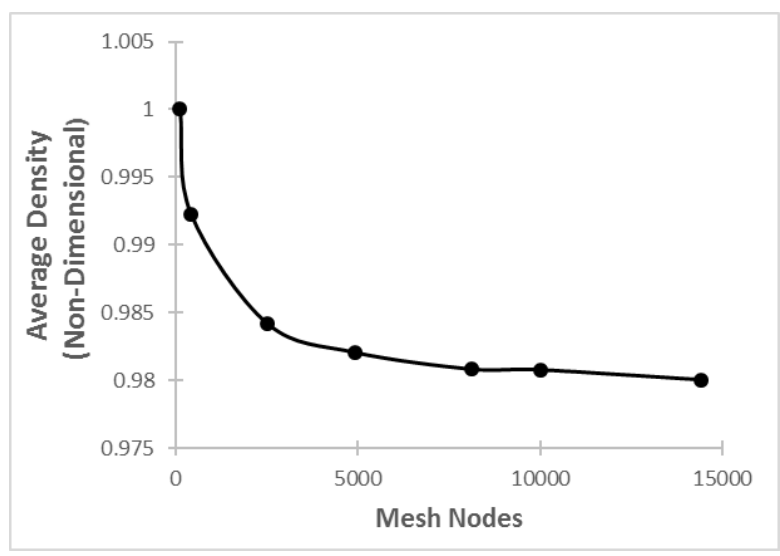

(b) Average Fluid Density

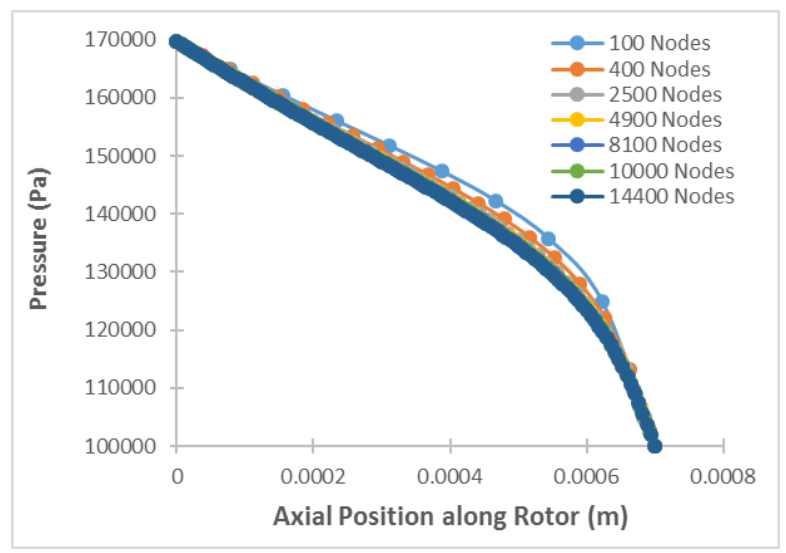

(c) Pressure

Fig. 4.1 Mesh Study Parameters

Figure 4.1(c) shows the pressure distribution along the rotor for each mesh. This demonstrates that a threshold exists for the utility of increasing the number of nodes used to calculate the solution. 


\subsection{Comparison with Experiments}

\subsubsection{Mass Flow Rate}

The ability of this code to predict mass flow rate can be assessed by comparing it with experimental test data. Four test cases were taken from the experimental data presented by Bayley and Long (Bayley \& Long, 1993) and simulated using the developed code. The operating parameters for these cases are listed in table 4.2.1 and the results are shown in Figure 4.2.1

\begin{tabular}{|c|c|c|}
\hline Inlet Pressure (Kpa) & Outlet Pressure (KPa) & Isothermal Temperature (c) \\
\hline 107.742 & 100 & 20 \\
\hline 189.032 & 100 & 20 \\
\hline 269.032 & 100 & 20 \\
\hline 399.355 & 100 & 20 \\
\hline
\end{tabular}

Table 4.2.1 Mass Flow Test Parameters

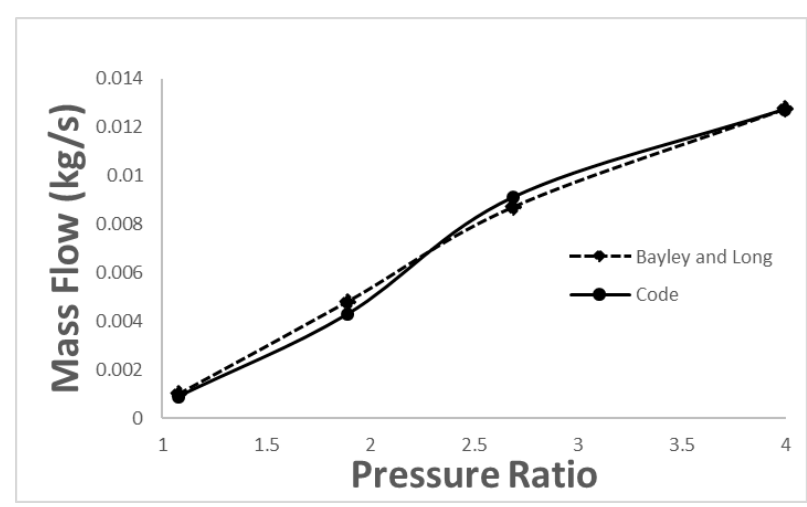

Fig. 4.2.1 Mass Flow Rate Comparison

The results shown above demonstrate that the difference between the results from the code and the results from the experiments tend to fluctuate over the range of pressure ratios. This can be attributed to the fact that the code does not model the bristle dynamics such as flutter, bending, and blow down. The bristle pack is only considered to be a stationary porous medium.

\subsubsection{Radial Pressure Distribution}

Figures 4.2.2(a)-(c) show the non-dimensional pressure variation in the radial direction just upstream of the back plate. $\mathrm{P}^{*}$ is the non-dimensional pressure and $\mathrm{Y}$ is the nondimensional radial coordinate. These parameters are determined in the exact same way as described in section 2.3.2. 


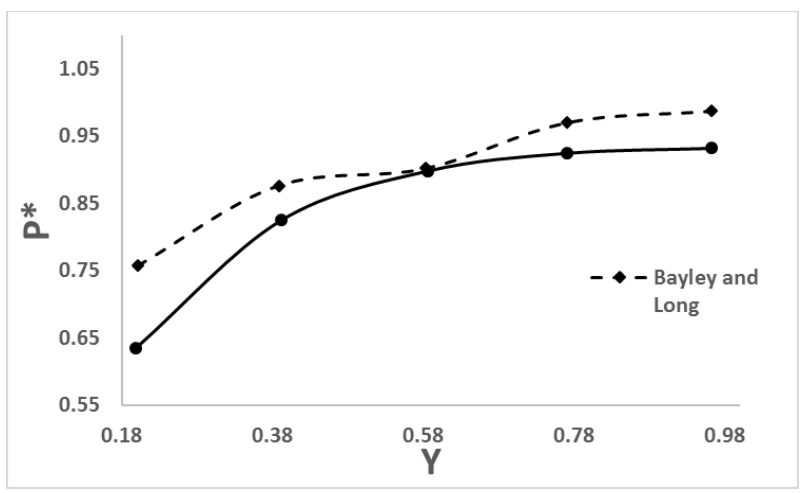

(a) Pressure ratio between 1.08 and 1.29

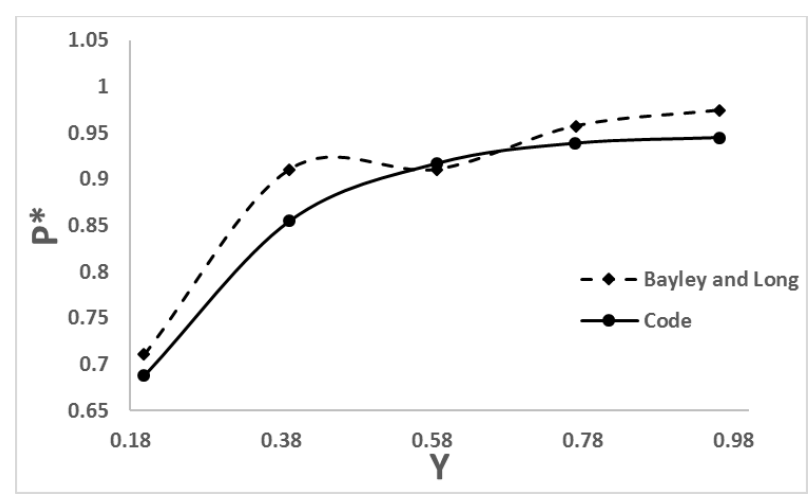

(b) Pressure ratio between 1.86 and 2.2

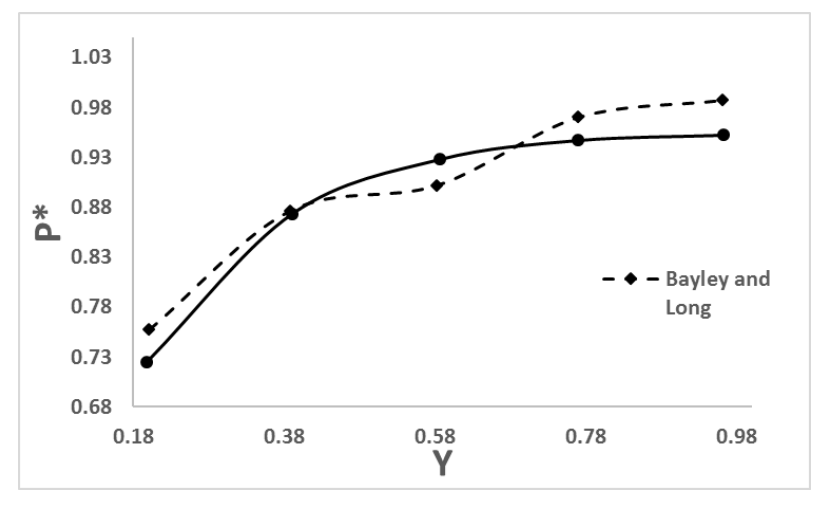

(c) Pressure ratio between 3.17 and 3.8

Fig. 4.2.2 Non-dimensional radial pressure distribution vs. non-dimensional radial coordinate. Results shown for three different ranges of pressure ratio.

The experimental results were collected by taking many samples of radial pressure distribution for a specific range of pressure ratios. The results from the code were generated by using the mean value of pressure ratio within the range. For example, the experimental results from the pressure ratio ranging between 1.08 and 1.29 is compared to results from the code where the pressure ratio was specified as 1.185 .

The code clearly does not capture some of the fluctuation of pressure along the radial direction of the back plate. It can also be seen that the radial pressure distribution predicted by the code becomes closer to the measured results at higher pressure ratios.

\subsubsection{Axial Pressure Distribution}

In the same way, the ability of the code to model axial distribution of pressure can be assessed by comparison with experiment. The tests conducted by Bayley and Long included measurements of axial pressure distribution along the shaft. Multiple tests were run for each range of pressure ratio. 


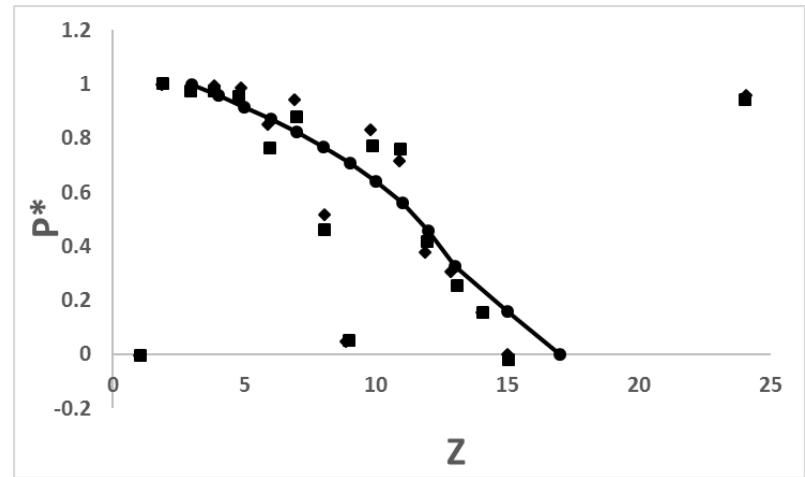

(a) Pressure ratio between 1.25 and 1.42

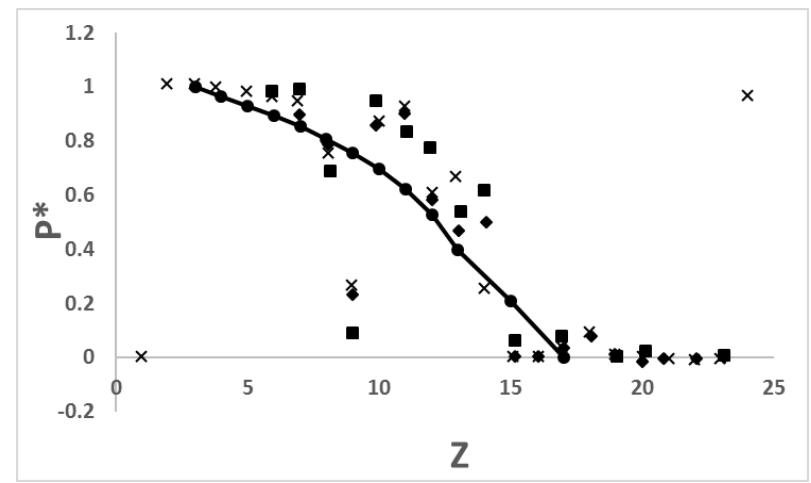

(b) Pressure ratio between 2.20 and 2.70

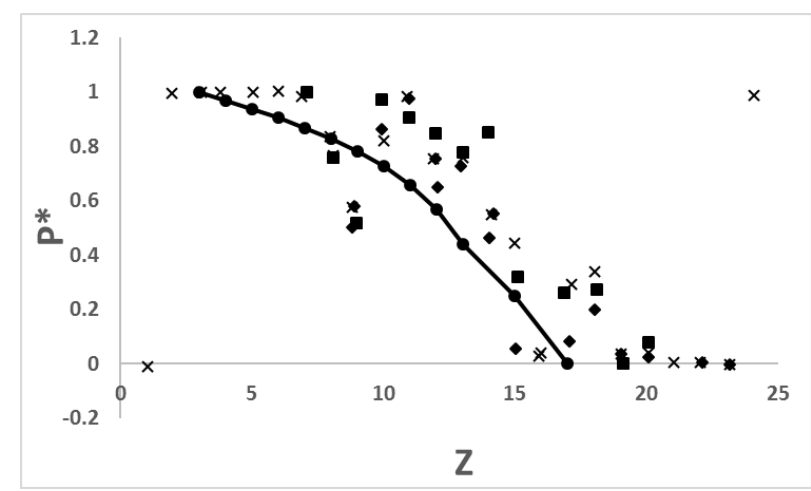

(c) Pressure ratio between 3.8 and 4.0

Fig. 4.2.3 Non-dimensional axial pressure distribution vs. non-dimensional axial coordinate. Results shown for three different ranges of pressure ratio.

The different symbols used in Figures 4.2.3(a)-(c) denote the results from different trials, and the solid line shows the prediction from the code. Once again, the pressure ratio specified for the code was taken to be the mean value of pressure ratio from the range used in experiments. The nondimensional axial coordinate $\mathrm{z}$ corresponds to the location of evenly spaced pressure tapings along the shaft. Further details on this parameter can be found in the reference (Bayley \& Long, 1993).

The results from this comparison show that the code tends to predict the pressure to be within the range of values determined from experiment. However, it also appears that the code begins to underestimate the pressure as pressure ratio between seal inlet and outlet is increased. More data at higher pressure ratio would be required to further test this trend. 


\subsection{Comparison with Commercial Software}

Further assessment of the performance of the code is possible with direct comparison to commercial-grade CFD software. A simple two-dimensional model was created to simulate flow through the bristle pack. This model used the same operating conditions, fluid properties and seal geometry as the developed code. The software used for comparison was ANSYS CFX.

Three sample cases were run using an inlet pressure of $128 \mathrm{KPa}$ and an outlet pressure of $100 \mathrm{KPa}$. The fluid was air modeled as an ideal gas, and the ratio of permeability in the $\mathrm{y}$ direction to permeability in the $x$ direction was different for each sample case. For the first case the ratio $\frac{\kappa_{y}}{\kappa_{x}}$ was equal to 1 , for the second case the ratio was 100 , and for the third case it was 1000. A comparison of the results are shown below:

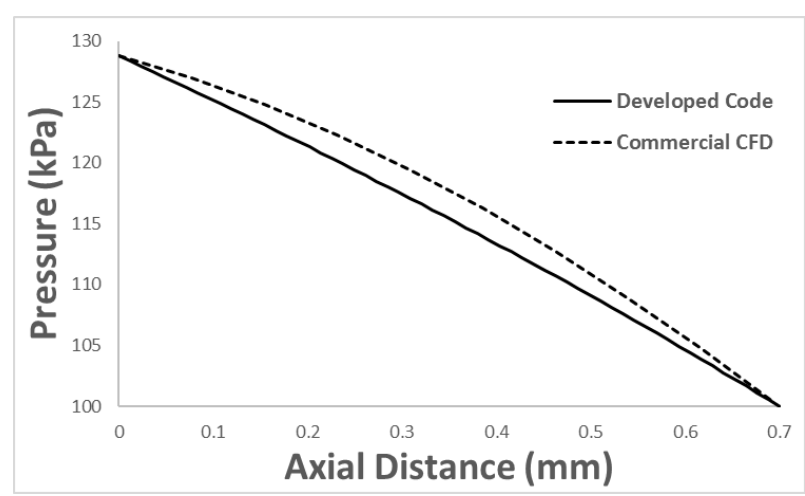

(a) Permeability Ratio = 1

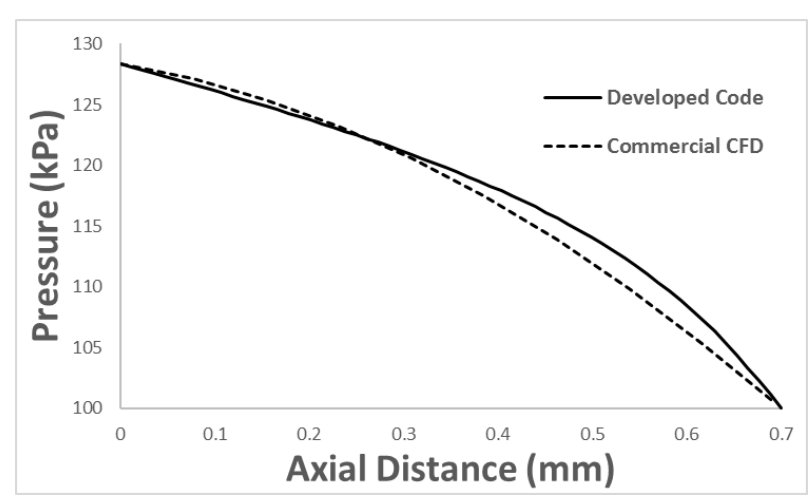

(b) Permeability Ratio $=100$

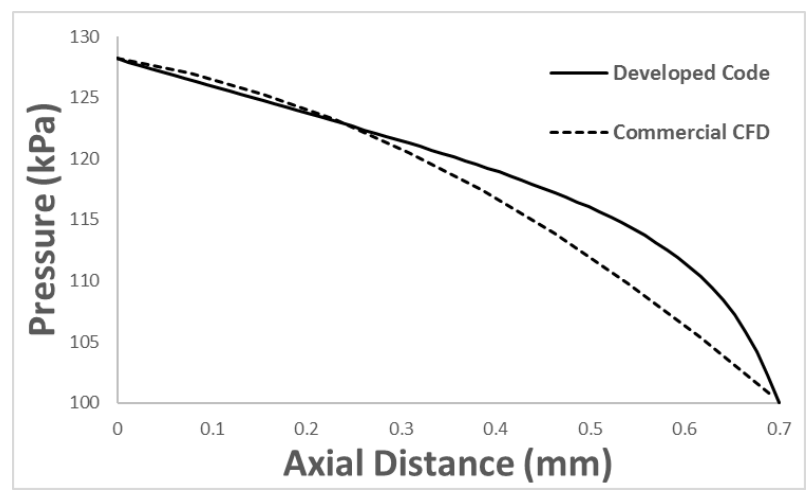

(c) Permeability Ratio $=1000$

Fig. 4.3.1 Axial pressure distribution comparison for three cases of permeability ratio. Note: data taken at radial height of $0.75 \mathrm{~mm}$ from rotor. 
Figures 4.3.1 (a)-(c) show the comparisons of axial pressure distribution for three different cases of permeability ratio. The axial pressure distribution shown is for a constant radial section $0.75 \mathrm{~mm}$ above the rotor surface. It is noteworthy that the disagreement between ANSYS CFX and the developed code shows some variation with permeability ratio.

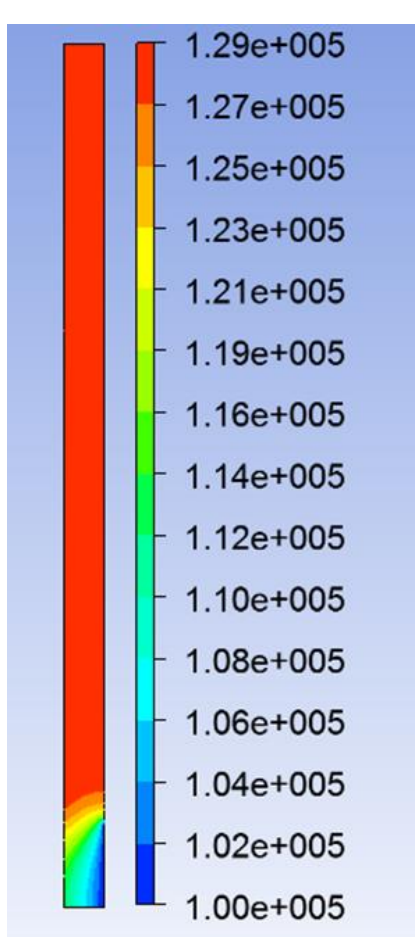

(a) ANSYS CFX, Permeability Ratio=1

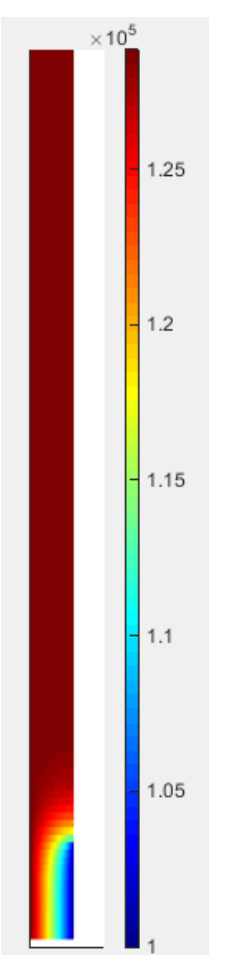

(b) Developed Code, Permeability Ratio=1 


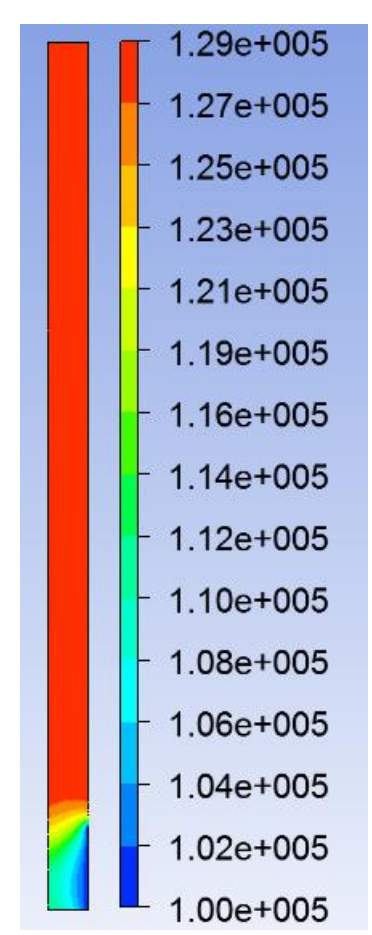

(a) ANSYS CFX, Permeability Ratio=10

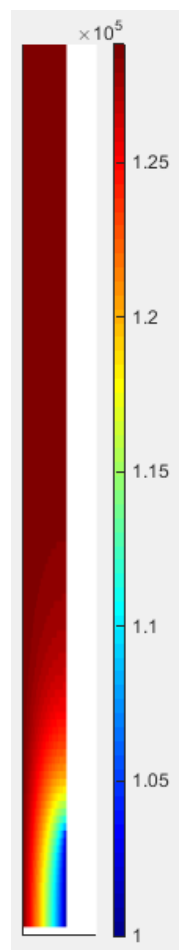

(b) Developed Code, Permeability Ratio=10

Fig. 4.3.2 Comparison of pressure distribution in the bristle pack. ANSYS CFX and the author's code.

Figures 4.3.2 shows comparisons between the predicted pressure distribution from ANSYS CFX and the pressure distribution predicted from the code described in this thesis. The pressure ratio used for these comparisons was chosen to be 1.29 and the axial permeability was $2.4 \times 10^{-11} \mathrm{~m}^{2}$. These values were selected because they are representative of standard parameters. It is again demonstrated that the results from this code are more dramatically affected by changes in the relative permeability ratio $\left(\frac{k_{y}}{k_{x}}\right)$ than the results from ANSYS CFX. 


\section{Chapter 5 \\ Conclusions and Future Work}

This study used CFD to assess the variation of axial permeability within the bristle pack of a brush seal. It was determined that the permeability of a standard brush seal varied by a percent difference of $75 \%$ when pressure ratio was varied from 1.2 to 4 . This result verified the expectation that axial permeability of a bristle pack can vary significantly over standard ranges of pressure ratio conditions.

In order to provide a simple and efficient solution for studying the performance of brush seals a new code was developed. This code used a two-dimensional finite difference scheme to discretize the governing equations for incompressible and slightly compressible flow through a porous domain. The theoretical performance of this scheme was analyzed and the limits of its applications were assessed.

Finally, the code was used to develop a brush seal model based on a seal design for which there is experimental data available in the literature. Predictions for pressure distribution and mass flow rate through the bristle pack were compared to experimental results and results from a commercial CFD software. The finite difference code shows good agreement with experimental results. The comparison to commercial CFD shows strong agreement for cases where the radial permeability is between 1 and 1000 times the axial permeability.

The governing two-dimensional equations used in this model were derived from simplifications which limit the application to cases in which flow behavior is only slightly compressible and inertial effects are small. Therefore, it should be acknowledged that the code developed in this thesis is a useful tool for estimating seal performance under conditions where flow is driven by an axial pressure gradient and inertial effects do not dominate flow behavior.

With this model available, engineers and scientists studying the design and performance of turbomachinery have yet another tool at their disposal. Flow rate can be calculated, pressure loadings on bristles can be estimated, and results can be obtained in a timely manner. 


\section{References}

\section{Bibliography}

Aksit, M. (2012). Brush Seal Dynamic Stiffness Behavior. Tuzla, Istanbu, Turkey: Sabanci University.

Bayley, F., \& Long, C. (1993). A Combined Experimental and Theoretical. ASME J. Eng. Gas Turbines and Power, 404-410.

Braun, M., Hendricks, R., \& Canacci, V. (1990). Flow Visualization in a Simulated Brush Seal. Proceedings of the Gas Turbine and Aeroengine Congress and Exposition. Brussles, Belgium.

Carlile, J., Hendricks, R., \& Yoder, D. (1993). Btush Seal Leakage Performance with Gaseous Working Fluids at Static and Low Rotor Speed Conditions. Journal of Engineering for Gas Turbines and Power, 397-403.

Chew, J., \& Hogg, S. (1997). Porosity Modeling of Brush Seals. Journal of Tribology, 769-775.

Chew, J., Lapworth, B., \& Millener, P. (1995). Mathematical modeling of brush seals. International Journal of Heat and Fluid Flow.

Childs, D. (1993). Turbomachinery Rotordynamics. John Wiley \& Sons.

Chilton, T., \& Colburn, A. (1931). Pressure Drop in Packed Tubes. Industrial and Engineering Chemistry, 913-919.

Cieślewicz, S. M. (2004). CFD-Simulations for Advanced Turbomachinery. Vienna, Austria: Institute for Thermodynamics and Energy Conversion.

Crudgington, P., Bowsher, A., \& Lloyd, D. (2009). Bristle Angle Effects on Brush Seal Contact Pressures. 45th AIAA/ASME/SAE/ASEE Joint Propulsion Conference \& Exhibit. Denver, Colorado.

Dogu, Y. (2005). Investigation of Brush Seal Flow Characteristics Using Bulk Porous Medium Approach. J. Eng. Gas Turbines Power 127(1), 136-144.

Ergun, S. (1952). Fluid flow through packed columns. Chem. Eng. Prog. 48.

Fellenstein, J., \& DellaCorte, C. (1995). A New Tribological Test for Candidate Brush Seal Material Evaluation. Proceedings of the Society of Tribologists and Lubrication Engineers Annual Meeting. Chicago, Illinois.

Greenberg, M. (1998). Advanced Engineering Mathematics, Second Edition. Upper Saddle River, New Jersey: Prentice_Hall.

Guo, P. (2012). Dependency of Tortuosity and Permeability of Porous Media on Directional Distribution of Pore Voids. Transport in Porous Media, 285-303. 
Hanzlik, H. J. (1931). "U.S. Patent No. US 1831242 A, Labyrinth Packing". Washington, DC: U.S. Patent and Trademark Office.

Hendricks, R. C., Griffin, T. A., R, K. T., Csavina, K. R., Pancholi, A., \& Sood, D. (1994). Relative Performance Comparison Between Baseline Labyrinth and Dual-Brush Compressor Discharge Seals in a T-700 Engine Test. Proceedings of the ASME International Gas Turbine and Aeroengine Congress and Exposition. The Hague, Netherlands.

Hendricks, R., \& Schlumberger, S. (1991). A Bulk Flow Model of a Brush Seal System. International Gas Turbine and Aeroengine Congress and Exposition. Orlando, Florida.

Jambhekar, V. A. (2011). Forchheimer Porous-media Flow Models -. Stuttgart, Germany: Universität Stuttgart - Institut für Wasser- und Umweltsystemmodellierung.

Li, J. (2012). Experimental and Numerical Investigations on the Leakage Flow Characteristics of the Labyrinth Brush Seal. Journal of Engineering for Gas Turbines and Power.

Li, J., \& Yang, J. (2012). Analytical Investigations on the Contact Force Between Bristle Packs and Shaft Surface of Brush Seals. Proceedings of ASME Turbo Expo. Copenhagen, Denmark.

Migliorini, P., Untaroiu, A. W., \& Allaire, P. E. (2012). A Computational Fluid Dynamics/Bulk-Flow Hybrid Method for Determining Rotordynamic Coefficients of Annular Gas Seals. Journal of Tribology134(2).

Nolen-Hoeksema, R. (2014). Defining Permeability: Flow Through Pores. Schlumberger Oilfield Review, 63-64.

Nordmann, R., \& Weiser, P. (1991). EVALUATION OF ROTORDYNAMIC COEFFICIENTS OF LOOK-THROUGH LABYRINTHS. Kaiserslautern, Germany: University of Kaiserslautern.

O'Neil, A. (1993). Experiments on the Performance of Single and Multiple Brush Seals at Engine Representative Conditions. Department of Engineering, University of Oxford.

Pastrana, R., Wolfe, C., Turnquist, N., \& ME, B. (2001). Improved Steam Turbine Leakage Control With a Brush Seal Design. 30th Turbomachinery Symposium, (p. 34).

Pekris, M., Franceschini, G., \& Gillespie, D. R. (2012). An Investigation of Flow, Mechanical, and Thermal Performance of Conventional and Pressure-Balanced Brush Seals. Proceedings of ASME Turbo Expo. Copenhagen, Denmark.

Pgachev, A., \& Helm, P. (2008). Calibration of porous medium models for brush seals. Proceedings of The Institution of Mechanical Engineers, Part A: J. Power and Energy 223(1), (pp. 83-91).

Qiu, B., \& Li, J. (2013). Numerical Investigations on the Heat Transfer Behavior of Brush Seals Using Combined Computational Fluid Dynamics and Finite Element Method. Journal of Heat Transfer, vol 135. 
Qiu, B., Li, J., \& Yan, X. (2014). Investigation Into the Flow Behavior of Multi-Stage Brush Seals. Journal of Power and Energy, 416-428.

Saber, E., \& Abdou, K. (2010). Advanced Seal Design for Rotating Machinery. American Journal of Scientific and Indistrial Research.

Suryanarayanan, S. (2009). Labyrinth Seal Leakage Equation. Texas A\&M.

Untaroiu, A., Liu, C., Migliorini, P. J., Wood, H. G., \& Untaroiu, C. (2014). Hole-Pattern Seals Performance Evaluation Using Computational Fluid Dynamics and Design of Experiments Techniques. Journal of Engineering for Gas Turbines and Power.

Vance, J. M. (2010). Machinery Vibration and Rotordynamics. Hoboken, N.J.: Wiley.

Williams, B., \& Flack, R. (1998). Calculation of Rotor Dynamic Coefficients for Labyrinth Seals. International Journal of Rotating Machinery.

Yoon, S. (2013). Control of Surge in Centrifugal Compressors by Active Magnetic Bearings. London: Springer-Verlag.

Young, D. M. (1971). Iterative Solution of Large Linear Systems. New York: Academic Press.

Young, D. M. (1971). Iterative Solution of Large Linear Systems. New York: Academic Press. 